\title{
An explicit solution for implicit time stepping in finite strain viscoelasticity.
}

\author{
A.V. Shutov*, R. Landgraf, J. Ihlemann \\ Chemnitz University of Technology, Department of Solid Mechanics, Str. d. \\ Nationen 62, D-09111 Chemnitz, Germany
}

\begin{abstract}
We consider the numerical treatment of one of the most popular finite strain models of the viscoelastic Maxwell body. This model is based on the multiplicative decomposition of the deformation gradient, combined with Neo-Hookean hyperelastic relations between stresses and elastic strains. The evolution equation is six dimensional. For the corresponding local initial value problem, a fully implicit integration procedure is considered, and a simple explicit update formula is derived. Thus, no local iterative procedure is required, which makes the numerical scheme more robust and efficient. The resulting integration algorithm is unconditionally stable and first order accurate. The incompressibility constraint of the inelastic flow is exactly preserved. A rigorous proof of the symmetry of the consistent tangent operator is provided. Moreover, some properties of the numerical solution, like invariance under the change of the reference configuration and positive energy dissipation within a time step, are discussed. Numerical tests show that, in terms of accuracy, the proposed integration algorithm is equivalent to the classical implicit scheme based on the exponential mapping. Finally, in order to check the stability of the algorithm numerically, a representative initial boundary value problem involving finite viscoelastic deformations is considered. An FEM solution of the representative problem using MSC.MARC is presented.
\end{abstract}

Key words: viscoelasticity, Maxwell fluid, finite strains, implicit time stepping, integration algorithm, Euler-backward method.

AMS Subject Classification: 74C20; 65L20; 76A10.

* Corresponding author. Tel.: +49-0-371-531-35024; fax: +49-0-371-531-23419.

Email address: alexey.shutov@mb.tu-chemnitz.de (A.V. Shutov). 


\section{Introduction}

Among idealized models of linear viscoelasticity, the so-called Maxwell fluid (MF) is commonly encountered in material modelling $[53,16,2]$. The one-dimensional rheological interpretation of this model is shown in Fig. 1a.1 A series of Maxwell elements connected in parallel [67] can be utilized to represent viscoelastic properties of polymers (Fig. 1b). In that case, the stresses acting in the Maxwell elements can be associated with overstresses [16]. Next, a slightly modified Maxwell element can be adopted to capture the nonlinear kinematic hardening in metals (Fig. 1c). In that case, the corresponding Maxwell stresses are interpreted as backstresses [39,3,66,7,57]. Moreover, within some phenomenological approaches to metal plasticity, the distortional hardening in metals can be captured using the modified MF $[58,60]$. Other groups of materials like shape memory alloys $[18,20]$ and biological tissues [8] can be modelled using MF. Some applications of MF to finite deformations of geological structures [61,49] and to fluid mechanics [1] are reported in the literature as well.

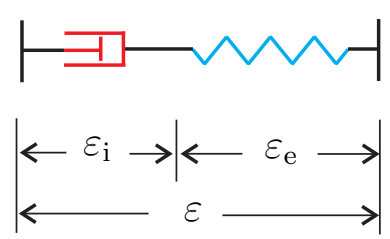

a)

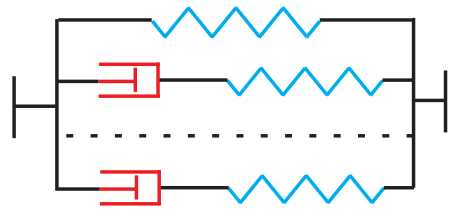

b)

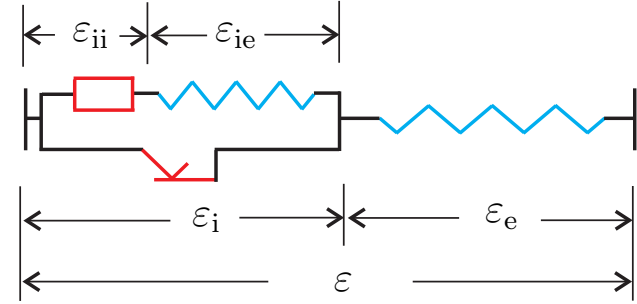

c)

Fig. 1. a) A one-dimensional Maxwell body consists of an elastic spring (Hooke body) coupled in series with a viscous dashpot (Newton body), b) Generalized Maxwell body, also known as Wiechert model (or Zener model in a special case) used for better description of viscoelastic properties, c) modified Schwedoff model used to represent nonlinear kinematic hardening.

In the finite strain range, numerous constitutive models of the MF exist (see, among others, $[37,27,33,48,46,47,23,4,51,1,16,17])$. Different variants were compared through numerical tests in $[10,34]$. In this paper we consider one of the most popular models of the MF. The corresponding constitutive equations are summarized in Section 2 of this work. The model under consideration is a special case of the finite strain viscoplasticity model proposed by Simo and Miehe [62], and it has the same structure as the well known model of associative elastoplasticity considered by Simo [63]. These models were developed within the framework of multiplicative inelasticity in combination with hyperelastic constitutive relations. The corresponding inelastic flow rule 2 is six dimensional since the inelastic spin plays no role due to elastic isotropy. A version of the MF which is equivalent to the version of Simo \& Miehe was considered later in material (Lagrangian) description by Lion [38]. This Lagrangian formulation was adopted in $[40,18,12,54,56,58]$. The spatial

$\overline{1}$ A two-dimensional rheological model of the Maxwell fluid and its modifications can be found in $[58,60]$.

2 In this paper, the evolution equation is referred to as "inelastic flow rule" in order to stress that the model is a special case of a viscoplastic model. 
(Eulerian) constitutive equations proposed by Simo \& Miehe were utilized later in the comprehensive study by Reese and Govindjee [51], and by many others (see, for instance, $[24,43,49,30,15,22,50,36])$.

In the modern literature on numerics, much attention is paid to general procedures which can be implemented to different types of constitutive relations in a straightforward manner. Because of their generality, such procedures are not always efficient being compared to algorithms which make use of the special structure of the underlying constitutive equations. Due to the high prevalence of the Simo \& Miehe version of the Maxwell fluid in material modelling, efficient and robust numerical integration of the underlying evolution equations is a challenging task. The main purpose of this paper is to report a new, simple, and efficient numerical procedure for this model.

Since the corresponding initial value problem is typically stiff, implicit time stepping methods should be implemented. For the Simo \& Miehe version of the MF, a discretized problem can be obtained using the operator split technique in combination with exponential mapping and formulation in principal axes as described in [63] and [51] (Eulerian approach). Alternatively, the evolution equation formulated on the reference configuration can be discretized as described in [11] (Lagrangian approach). In both cases, a system of nonlinear algebraic equations is obtained, and a local iterative procedure is usually implemented to resolve the resulting nonlinear problem (see, among others, $[51,44,12,13,49,19,30,55,66,15,22,50,36])$. Obviously, such iterative procedures can slow down the entire FEM simulation. This problem may become especially important if globally explicit FEM is considered 3 This publication is dealing with first-order accurate methods only. For the discussion concerning the application of higher order methods, the reader is referred to $[52,14,5,6]$.

In order to speed up the FEM computations, much attention was paid to the construction of closed form solutions for implicit schemes. For instance, a simplified flow rule under the assumption of small elastic strains was considered by Simo and Miehe [62] in order to get an explicit update formula for the local implicit time stepping procedure. For the same reason, another simplification of the flow rule in case of small elastic strains was considered by Reese and Govondjee [51]. This simplified version was implemented later in [26]. Unfortunately, the simplifying assumption of small elastic strains is not valid for many materials like plastics, rubber, biological tissues etc. Moreover, if the modified Maxwell body is used to capture nonlinear kinematic hardening in metals, a general finite strain version of the model must be utilized as well 4 Another approach to closed form solution is based on special assumptions concerning the energy storage. In particular, a quadratic logarithmic strain energy (so-called Hencky strain energy) can be assumed in order to simplify the numerical treatment of the material model [41]. Unfortunately, this

3 In the case of explicit FEM, the evaluation of the material routine at each point of Gauss integration constitutes the major part of the overall computational effort.

4 In fact, although the elastic strains in metals are typically small ( $\varepsilon_{\mathrm{e}} \rightarrow 0$ in Fig. 1c), the conservative part ( $\varepsilon_{\mathrm{ie}}$ in Fig. 1c) of the inelastic strain may become finite. 
assumption would yield unrealistic results in case of large elastic strains. Thus, again, the applicability area is limited to moderate elastic strains. In this work, a simple explicit update formula is presented for the original finite strain version with Neo-Hookean potential. Interestingly, this explicit solution for the general case is even more compact and simple than the solutions presented in [62] and [51] for the special case of small elastic strains or the solution in [41] for quadratic logarithmic strain energy. For the new method, the computational effort per single time step is even smaller than the effort required within the explicit time stepping.

The inelastic flow is assumed to be incompressible, and the algorithm presented in this work preserves this incompressibility constraint. A classical model of finite strain viscoplasticity which contains the Simo \& Miehe version of the MF was considered in [56]. As it was shown in [56], the exact solution to the initial value problem is exponentially stable with respect to small perturbations of the initial data, if the incompressibility constraint is not violated. For such material models, the numerical schemes which exactly preserve the incompressibility are advantageous due to the suppressed error accumulation [56]. This theoretical result is confirmed by numerical tests presented in the current paper.

Dealing with the constitutive equations written in Lagrangian form, it can be shown that they are invariant under the isochoric change of the reference configuration [59]. The same invariance property can be formulated for the numerical solution as well. Obviously, the numerical algorithms which exactly retain this invariance property are advantageous. In this work, it is proved that the advocated algorithm retains the invariance of the solution.

We conclude this introduction with a few words regarding notation. Throughout this article, bold-faced symbols denote first- and second-rank tensors in $\mathbb{R}^{3}$. A coordinate-free tensor formalism is used in this work [25,55]. In this work, $\mathbf{1}$ stands for the second-rank identity tensor. The deviatoric part of a tensor is defined as $\mathbf{A}^{\mathrm{D}}:=\mathbf{A}-\frac{1}{3} \operatorname{tr}(\mathbf{A}) \mathbf{1}$, where $\operatorname{tr}(\mathbf{A})$ stands for the trace. The material time derivative is denoted by $\operatorname{dot}: \frac{d}{d t} \mathbf{A}=\dot{\mathbf{A}}$. The overline $\overline{(\cdot)}$ denotes the unimodular part of a tensor such that $\overline{\mathbf{A}}=(\operatorname{det} \mathbf{A})^{-1 / 3} \mathbf{A}$. The inverse of transposed tensor is denoted by $\mathbf{A}^{-\mathrm{T}}$. The positive definiteness of a tensor $\mathbf{A}$ is symbolically denoted by $\mathbf{A}>0$.

\section{System of constitutive equations}

\subsection{Lagrangian formulation}

Let us consider a finite strain model of Maxwell fluid. This model is covered as a special case by the viscoplasticity model presented by Simo and Miehe [62]. The Lagrangian formulation of the model follows the presentation of Lion [38]. We start with the multi-

plicative decomposition of the deformation gradient $\mathbf{F}$ into the elastic part $\hat{\mathbf{F}}_{\mathrm{e}}$ and the 
inelastic part $\mathbf{F}_{\mathrm{i}}$

$$
\mathbf{F}=\hat{\mathbf{F}}_{\mathrm{e}} \mathbf{F}_{\mathrm{i}}
$$

Along with the well-known right Cauchy-Green tensor $\mathbf{C}=\mathbf{F}^{\mathrm{T}} \mathbf{F}$, we introduce the inelastic right Cauchy-Green tensor as

$$
\mathbf{C}_{\mathrm{i}}=\mathbf{F}_{\mathrm{i}}^{\mathrm{T}} \mathbf{F}_{\mathrm{i}}
$$

The elastic right Cauchy-Green tensor $\hat{\mathbf{C}}_{\mathrm{e}}$ and the elastic Green tensor $\hat{\boldsymbol{\Gamma}}_{\mathrm{e}}$ are defined by

$$
\hat{\mathbf{C}}_{\mathrm{e}}:=\hat{\mathbf{F}}_{\mathrm{e}}^{\mathrm{T}} \hat{\mathbf{F}}_{\mathrm{e}}, \quad \hat{\boldsymbol{\Gamma}}_{\mathrm{e}}=\frac{1}{2}\left(\hat{\mathbf{C}}_{\mathrm{e}}-\mathbf{1}\right) .
$$

Next, we introduce the inelastic velocity gradient $\hat{\mathbf{L}}_{\mathrm{i}}$ and the covariant Oldroyd derivative (with respect to the intermediate configuration)

$$
\hat{\mathbf{L}}_{\mathrm{i}}=\dot{\mathbf{F}}_{\mathrm{i}} \mathbf{F}_{\mathrm{i}}^{-1}, \quad \stackrel{\Delta}{(\cdot)}=\frac{d}{d t}(\cdot)+\hat{\mathbf{L}}_{\mathrm{i}}^{\mathrm{T}}(\cdot)+(\cdot) \hat{\mathbf{L}}_{\mathrm{i}}
$$

The inelastic Almansi strain tensor $\hat{\boldsymbol{\Gamma}}_{\mathrm{i}}$ and the inelastic strain rate tensor $\hat{\mathbf{D}}_{\mathrm{i}}$ are defined through

$$
\hat{\boldsymbol{\Gamma}}_{\mathrm{i}}:=\frac{1}{2}\left(\mathbf{1}-\mathbf{F}_{\mathrm{i}}^{-\mathrm{T}} \mathbf{F}_{\mathrm{i}}^{-1}\right), \quad \hat{\mathbf{D}}_{\mathrm{i}}=\frac{1}{2}\left(\hat{\mathbf{L}}_{\mathrm{i}}+\hat{\mathbf{L}}_{\mathrm{i}}^{\mathrm{T}}\right) .
$$

After some straightforward computations (cf. [16]), one gets

$$
\hat{\mathbf{D}}_{\mathrm{i}}=\hat{\boldsymbol{\Gamma}}_{\mathrm{i}} .
$$

Let $\mathbf{T}$ be the Cauchy stress tensor. The Kirchhoff stress tensor $\mathbf{S}$, the 2nd Piola-Kirchhoff tensor $\hat{\mathbf{S}}$ operating on the intermediate configuration and the 2nd Piola-Kirchhoff tensor $\tilde{\mathbf{T}}$ operating on the reference configuration are defined through

$$
\mathbf{S}:=(\operatorname{det} \mathbf{F}) \mathbf{T}, \quad \hat{\mathbf{S}}:=\hat{\mathbf{F}}_{\mathrm{e}}^{-1} \mathbf{S} \hat{\mathbf{F}}_{\mathrm{e}}^{-\mathrm{T}}, \quad \tilde{\mathbf{T}}:=\mathbf{F}^{-1} \mathbf{S} \mathbf{F}^{-\mathrm{T}} .
$$

Let $\psi$ be the free energy per unit mass. It is postulated in the Neo-Hookean form as

$$
\rho_{\mathrm{R}} \psi\left(\hat{\mathbf{C}}_{\mathrm{e}}\right)=\frac{\mu}{2}\left(\operatorname{tr} \overline{\hat{\mathbf{C}}_{\mathrm{e}}}-3\right) .
$$

Here, $\rho_{\mathrm{R}}>0$ stands for the mass density with respect to the reference configuration; $\mu \geq 0$ is the shear modulus. Next, noting that $\hat{\mathbf{C}}_{\mathrm{e}}=\mathbf{1}+2 \hat{\boldsymbol{\Gamma}}_{\mathrm{e}}$, a hyperelastic stress-strain relation is introduced on the intermediate configuration:

$$
\hat{\mathbf{S}}=\rho_{\mathrm{R}} \frac{\partial \psi\left(\mathbf{1}+2 \hat{\boldsymbol{\Gamma}}_{\mathrm{e}}\right)}{\partial \hat{\boldsymbol{\Gamma}}_{\mathrm{e}}} .
$$

Isothermal processes are considered in this study. The Clausius-Duhem inequality requires that the specific internal dissipation $\delta_{\mathrm{i}}$ remains non-negative (see [16])

$$
\delta_{\mathrm{i}}:=\frac{1}{\rho_{\mathrm{R}}} \tilde{\mathbf{T}}: \dot{\mathbf{E}}-\dot{\psi} \geq 0,
$$


where $\mathbf{E}:=\frac{1}{2}(\mathbf{C}-\mathbf{1})$ stands for the Green strain tensor. Using (3) and taking the isotropy of the free energy function into account, this inequality is reduced to

$$
\rho_{\mathrm{R}} \delta_{\mathrm{i}}=\left(\hat{\mathbf{C}}_{\mathrm{e}} \hat{\mathbf{S}}\right): \stackrel{\Delta}{\hat{\boldsymbol{\Gamma}}_{\mathrm{i}} \geq 0}
$$

An evolution equation is postulated so that inequality (5) holds for arbitrary mechanical loadings (cf. [38])

$$
\hat{\boldsymbol{\Gamma}}_{\mathrm{i}}=\frac{1}{2 \eta}\left(\hat{\mathbf{C}}_{\mathrm{e}} \hat{\mathbf{S}}\right)^{\mathrm{D}},
$$

where $\eta \geq 0$ is a material parameter (Newtonian viscosity) 5 In view of (1), an equivalent formulation of this flow rule is given by

$$
\hat{\mathbf{D}}_{\mathrm{i}}=\frac{1}{2 \eta}\left(\hat{\mathbf{C}}_{\mathrm{e}} \hat{\mathbf{S}}\right)^{\mathrm{D}}
$$

Note that this flow rule is six dimensional since the rotational part skew $\left(\hat{\mathbf{L}}_{\mathrm{i}}\right)$ drops out from the constitutive relations due to the elastic isotropy. Moreover, $\operatorname{since} \operatorname{tr}\left(\hat{\boldsymbol{\Gamma}}_{\mathrm{i}}\right)=\operatorname{tr}\left(\hat{\mathbf{D}}_{\mathrm{i}}\right)=0$, the inelastic flow described by (6) in incompressible.

In order to simplify the numerical treatment of the model, the constitutive equations can be transformed to the reference configuration. In particular, the free energy takes the form

$$
\psi=\psi\left(\mathbf{C C}_{\mathrm{i}}^{-1}\right)=\frac{\mu}{2 \rho_{\mathrm{R}}}\left(\operatorname{tr} \overline{\mathbf{C C}_{\mathrm{i}}^{-1}}-3\right) .
$$

Using (2) and (3), one gets for the 2nd Piola-Kirchhoff stress tensor

$$
\tilde{\mathbf{T}}=\left.2 \rho_{\mathrm{R}} \frac{\partial \psi\left(\mathbf{C C}_{\mathrm{i}}^{-1}\right)}{\partial \mathbf{C}}\right|_{\mathbf{C}_{\mathrm{i}}=\text { const }} .
$$

Substituting (8) into this relation, one gets

$$
\tilde{\mathbf{T}}=\mu \mathbf{C}^{-1}\left(\overline{\mathbf{C}} \mathbf{C}_{\mathrm{i}}^{-1}\right)^{\mathrm{D}}
$$

Next, we note that

$$
\operatorname{tr}\left(\hat{\mathbf{C}}_{\mathrm{e}} \hat{\mathbf{S}}\right)=\operatorname{tr}(\mathbf{C} \tilde{\mathbf{T}})
$$

Thus, the pull-back of the deviatoric part of the Mandel tensor $\hat{\mathbf{C}}_{\mathrm{e}} \hat{\mathbf{S}}$ is given by

$$
\mathbf{F}_{\mathrm{i}}^{\mathrm{T}}\left(\hat{\mathbf{C}}_{\mathrm{e}} \hat{\mathbf{S}}\right)^{\mathrm{D}} \mathbf{F}_{\mathrm{i}}=\mathbf{C} \tilde{\mathbf{T}} \mathbf{C}_{\mathrm{i}}-\frac{1}{3} \operatorname{tr}\left(\hat{\mathbf{C}}_{\mathrm{e}} \hat{\mathbf{S}}\right) \mathbf{C}_{\mathrm{i}} \stackrel{(5)}{=}(\mathbf{C} \tilde{\mathbf{T}})^{\mathrm{D}} \mathbf{C}_{\mathrm{i}}
$$

$\overline{5}$ In some works, the viscosity $\eta$ is replaced by the parameter $\varkappa>0$ such that $\varkappa=1 / \eta$. Furthermore, the relaxation time can be introduced by $\tau=\eta / \mu$. The case of process-dependent viscosity was considered, among others, in [37,33,38,57,31]. 
Using this relation in combination with the evolution equation (6), we get

$$
\dot{\mathbf{C}}_{\mathrm{i}}=2 \mathbf{F}_{\mathrm{i}}^{\mathrm{T}} \stackrel{\Delta}{\hat{\boldsymbol{\Gamma}}_{\mathrm{i}}} \mathbf{F}_{\mathrm{i}} \stackrel{(6)}{=} \frac{1}{\eta} \mathbf{F}_{\mathrm{i}}^{\mathrm{T}}(\hat{\mathbf{C}} \hat{\mathrm{e}} \hat{\mathbf{S}})^{\mathrm{D}} \mathbf{F}_{\mathrm{i}} \stackrel{(5)}{=} \frac{1}{\eta}(\mathbf{C} \tilde{\mathbf{T}})^{\mathrm{D}} \mathbf{C}_{\mathrm{i}} .
$$

Taking (9) into account, we have

$$
\dot{\mathbf{C}}_{\mathrm{i}}=\frac{\mu}{\eta}\left(\overline{\mathbf{C}} \mathbf{C}_{\mathrm{i}}^{-1}\right)^{\mathrm{D}} \mathbf{C}_{\mathrm{i}}
$$

The system of constitutive equations (9) and (10) is closed by specifying initial condinions

$$
\left.\mathrm{C}_{\mathrm{i}}\right|_{t=t^{0}}=\mathbf{C}_{\mathrm{i}}^{0} \text {. }
$$

Note that the exact solution to the evolution equation (10) has the following geometric property

$$
\mathbf{C}_{\mathrm{i}}(t) \in \mathbb{M} \quad \text { if } \quad \mathbf{C}_{\mathrm{i}}^{0} \in \mathbb{M} \text {, }
$$

where the manifold $\mathbb{M}$ is a set of symmetric unimodular tensors

$$
\mathbb{M}:=\{\mathbf{A} \in S y m: \operatorname{det} \mathbf{A}=1\} .
$$

It follows from (12) that $\mathbf{C}_{\mathrm{i}}$ remains positive definite if $\mathbf{C}_{\mathrm{i}}^{0}>0,6$

Remark 1 Interestingly, an evolution equation in the form (10) was presented for an alternative model of the MF by other authors (see equation (10.147) in [16]), although the flow rule considered on the intermediate configuration is given by $\hat{\mathbf{D}}_{\mathrm{i}}=\frac{1}{\eta} \hat{\mathbf{S}}$, which differs essentially from the flow rule (7). Since both evolution equations coincide, the approach advocated in this paper can be applied to the version presented in [16] without any modifications whatsoever.

\subsection{Eulerian formulation}

Now let us check that the model presented in this section coincides with the model of Simo and Miehe [62], formulated within the Eulerian approach. Here, elastic isotropy is considered. First, note that

$$
2 \hat{\mathbf{D}}_{\mathrm{i}}=\mathbf{F}_{\mathrm{i}}^{-\mathrm{T}} \dot{\mathbf{C}}_{\mathrm{i}} \mathbf{F}_{\mathrm{i}}^{-1} \text {. }
$$

Due to the elastic isotropy, according to the evolution equation (7), $\hat{\mathbf{D}}_{\mathrm{i}}$ commutes with $\hat{\mathbf{C}}_{\mathrm{e}}$. In particular, one gets from (7)

$$
\hat{\mathbf{C}}_{\mathrm{e}} 2 \hat{\mathbf{D}}_{\mathrm{i}} \hat{\mathbf{C}}_{\mathrm{e}}^{-1}=\frac{1}{\eta}\left(\hat{\mathbf{C}}_{\mathrm{e}} \hat{\mathbf{S}}\right)^{\mathrm{D}}
$$

$\overline{6}$ The eigenvalues of $\mathbf{C}_{\mathrm{i}}$ are continuous functions of time. Therefore, if all of the eigenvalues were positive at some time instance and their product remains constant in time, then they remain positive. 
Substituting (13) into (14) and taking into account that $\frac{d}{d t}\left(\mathbf{C}_{\mathrm{i}}^{-1}\right)=-\mathbf{C}_{\mathrm{i}}^{-1} \dot{\mathbf{C}}_{\mathrm{i}} \mathbf{C}_{\mathrm{i}}^{-1}$ one gets

$$
-\hat{\mathbf{F}}_{\mathrm{e}}^{\mathrm{T}} \mathbf{F} \frac{d}{d t}\left(\mathbf{C}_{\mathrm{i}}^{-1}\right) \mathbf{F}^{\mathrm{T}} \hat{\mathbf{F}}_{\mathrm{e}}^{-\mathrm{T}} \hat{\mathbf{F}}_{\mathrm{e}}^{-1} \hat{\mathbf{F}}_{\mathrm{e}}^{-\mathrm{T}}=\frac{1}{\eta}\left(\hat{\mathbf{C}}_{\mathrm{e}} \hat{\mathbf{S}}\right)^{\mathrm{D}}
$$

For what follows, we introduce the elastic left Cauchy-Green tensor $\hat{\mathbf{B}}_{\mathrm{e}}=\hat{\mathbf{F}}_{\mathrm{e}} \hat{\mathbf{F}}_{\mathrm{e}}^{\mathrm{T}}$, and note that

$$
\mathbf{S}^{\mathrm{D}}=\hat{\mathbf{F}}_{\mathrm{e}}^{-\mathrm{T}}\left(\hat{\mathbf{C}}_{\mathrm{e}} \hat{\mathbf{S}}\right)^{\mathrm{D}} \hat{\mathbf{F}}_{\mathrm{e}}^{\mathrm{T}}
$$

Multiplying (15) with $\hat{\mathbf{F}}_{\mathrm{e}}^{-\mathrm{T}}$ from the left and with $\hat{\mathbf{F}}_{\mathrm{e}}^{\mathrm{T}}$ from the right one gets using (2.2)

$$
-\mathbf{F} \frac{d}{d t}\left(\mathbf{C}_{\mathrm{i}}^{-1}\right) \mathbf{F}^{\mathrm{T}} \hat{\mathbf{B}}_{\mathrm{e}}^{-1}=\frac{1}{\eta} \mathbf{S}^{\mathrm{D}}
$$

By introducing the covariant Oldroyd rate 7

$$
\mathfrak{O}(\mathbf{A})=\mathscr{L}_{v}(\mathbf{A}):=\mathbf{F} \frac{d}{d t}\left(\mathbf{F}^{-1} \mathbf{A} \mathbf{F}^{-\mathrm{T}}\right) \mathbf{F}^{\mathrm{T}}=\dot{\mathbf{A}}-\mathbf{L} \mathbf{A}-\mathbf{A L}^{\mathrm{T}}
$$

one gets

$$
\mathfrak{O}\left(\hat{\mathbf{B}}_{\mathrm{e}}\right)=\mathscr{L}_{v}\left(\hat{\mathbf{B}}_{\mathrm{e}}\right)=\mathbf{F} \frac{d}{d t}\left(\mathbf{C}_{\mathrm{i}}^{-1}\right) \mathbf{F}^{\mathrm{T}} .
$$

Thus, the flow rule (16) takes the well-known form

$$
-\mathscr{L}_{v}\left(\hat{\mathbf{B}}_{\mathrm{e}}\right) \hat{\mathbf{B}}_{\mathrm{e}}^{-1}=\frac{1}{\eta} \mathbf{S}^{\mathrm{D}}
$$

This equation was covered as a special case by Simo and Miehe [62] (see equations (2.19a) and (2.26) in [62]) as well as by Reese and Govindjee [51]. In case of the Neo-Hookean potential (8), we have $\mathbf{S}=\mathbf{S}^{\mathrm{D}}=\mu\left(\hat{\mathbf{B}}_{\mathrm{e}}\right)^{\mathrm{D}}$, and the evolution equation (17) is reduced to

$$
-\mathscr{L}_{v}\left(\hat{\mathbf{B}}_{\mathrm{e}}\right) \hat{\mathbf{B}}_{\mathrm{e}}^{-1}=\frac{\mu}{\eta}\left(\overline{\hat{\mathbf{B}}_{\mathrm{e}}}\right)^{\mathrm{D}}
$$

\section{Time stepping algorithm}

\subsection{Explicit update formula in Lagrangian formulation}

Let us consider a typical time interval $\left(t_{n}, t_{n+1}\right)$ with $\Delta t:=t_{n+1}-t_{n}>0$. By ${ }^{n} \mathbf{C}_{\mathrm{i}}$ and ${ }^{n+1} \mathbf{C}_{\mathrm{i}}$ we denote numerical solutions respectively at $t_{n}$ and $t_{n+1}$. Suppose that the deformation gradient ${ }^{n+1} \mathbf{F}$ at time instance $t_{n+1}$ is known, and ${ }^{n} \mathbf{C}_{\mathrm{i}} \in \mathbb{M}$ is given. The unknown ${ }^{n+1} \mathbf{C}_{\mathrm{i}} \in \mathbb{M}$ is estimated as the unimodular part of the solution provided by

7 In order to sress that this Oldroyd rate is also a Lee derivative, it can be denoted by $\mathscr{L}_{v}$. 
the classical Euler-backward method (EBM). In other words, let ${ }^{n+1} \mathbf{C}_{\mathrm{i}}^{\mathrm{EBM}}$ be the EBM solution. After the subsequent correction, the solution is given by

$$
{ }^{n+1} \mathbf{C}_{\mathrm{i}}:=\overline{{ }^{n+1} \mathbf{C}_{\mathrm{i}}^{\mathrm{EBM}}}=\left(\operatorname{det}\left({ }^{n+1} \mathbf{C}_{\mathrm{i}}^{\mathrm{EBM}}\right)\right)^{-1 / 3}{ }^{n+1} \mathbf{C}_{\mathrm{i}}^{\mathrm{EBM}} .
$$

Let us derive this solution. First, adopting EBM, the discretized version of (10) reads

$$
{ }^{n+1} \mathbf{C}_{\mathrm{i}}^{\mathrm{EBM}}={ }^{n} \mathbf{C}_{\mathrm{i}}+\frac{\Delta t \mu}{\eta}\left({ }^{n+1} \overline{\mathbf{C}}\left({ }^{n+1} \mathbf{C}_{\mathrm{i}}^{\mathrm{EBM}}\right)^{-1}\right)^{\mathrm{D}}{ }^{n+1} \mathbf{C}_{\mathrm{i}}^{\mathrm{EBM}},
$$

where ${ }^{n+1} \mathbf{C}={ }^{n+1} \mathbf{F}^{\mathrm{T}}{ }^{n+1} \mathbf{F}$ is given. Introducing abbreviation

$$
\beta:=\frac{1}{3} \frac{\Delta t \mu}{\eta} \operatorname{tr}\left({ }^{n+1} \overline{\mathbf{C}}\left({ }^{n+1} \mathbf{C}_{\mathrm{i}}^{\mathrm{EBM}}\right)^{-1}\right),
$$

(20) can be rewritten as

$$
{ }^{n+1} \mathbf{C}_{\mathrm{i}}^{\mathrm{EBM}}={ }^{n} \mathbf{C}_{\mathrm{i}}+\frac{\Delta t \mu}{\eta}{ }^{n+1} \overline{\mathbf{C}}-\beta^{n+1} \mathbf{C}_{\mathrm{i}}^{\mathrm{EBM}}
$$

Thus, obviously

$$
{ }^{n+1} \mathbf{C}_{\mathrm{i}}^{\mathrm{EBM}}=\frac{1}{1+\beta}\left({ }^{n} \mathbf{C}_{\mathrm{i}}+\frac{\Delta t \mu}{\eta}{ }^{n+1} \overline{\mathbf{C}}\right) .
$$

Finally, noting that $\frac{1}{1+\beta} \mathbf{A}=\overline{\mathbf{A}}$ for any $\mathbf{A}$, and substituting (22) into (19), the following explicit update formula is obtained

$$
{ }^{n+1} \mathbf{C}_{\mathrm{i}}=\overline{{ }^{n} \mathbf{C}_{\mathrm{i}}+\frac{\Delta t \mu}{\eta}{ }^{n+1} \overline{\mathbf{C}}}
$$

Remark 2 Note that the solution ${ }^{n+1} \mathbf{C}_{\mathrm{i}}$ is obtained directly, without computing ${ }^{n+1} \mathbf{C}_{\mathrm{i}}^{\mathrm{EBM}}$. On the other hand, a simple explicit update formula can be derived for EBM as well (see Appendix A).

\subsection{Properties of the algorithm in Lagrangian formulation}

Recall that the exact solution to the evolution equation (10) has under proper initial conditions the geometric property $\mathbf{C}_{\mathrm{i}} \in \mathbb{M}$. The numerical algorithms which exactly preserve this property are referred to as geometric integrators. It was shown in [56] that such integrators allow to suppress the error accumulation, which is especially important for the simulation of "long" processes. Therefore, geometric integrators are advantageous. Obviously, the geometric property (12) is exactly retained by the numerical solution (23).

Remark 3 Observe that the method (19) corresponds to EBM with a subsequent correction. Another modification of the classical EBM was considered by Helm [19] in order 
to obtain a geometric integrator. Furthermore, in the paper by Vladimirov et al. [66], two other modifications were considered and the plastic incompressibility was enforced by introducing the additional equation $\operatorname{det} \mathbf{C}_{\mathrm{i}}=1$ with an additional unknown scalar variable. In contrast to the explicit update formula (23), a local iterative procedure was implemented in [19] and [66].

Another property of the algorithm is as follows. As already mentioned in Section 2.1, the exact solution $\mathbf{C}_{\mathrm{i}}$ must be positive definite. Since ${ }^{n} \mathbf{C}_{\mathrm{i}}$ and ${ }^{n+1} \overline{\mathbf{C}}$ are positive definite, the numerical solution ${ }^{n+1} \mathbf{C}_{i}$ given by (23) is positive definite as well. Indeed, the sum of two positive definite tensors is positive definite, and the projection operator $\overline{(\cdot)}$ retains this property. Furthermore, due to the positive definiteness, $\operatorname{det}\left({ }^{n} \mathbf{C}_{\mathrm{i}}+\frac{\Delta t \mu}{\eta}{ }^{n+1} \mathbf{C}\right)>0$. Thus, the unimodular part of $\left({ }^{n} \mathbf{C}_{\mathrm{i}}+\frac{\Delta t \mu n+1}{\eta} \overline{\mathbf{C}}\right)$ in (23) is well defined for all $\Delta t \geq 0$ and ${ }^{n+1} \mathbf{C}_{\mathrm{i}}$ it is a smooth function of $\Delta t$. For $\Delta t \geq 0$, the solution ${ }^{n+1} \mathbf{C}_{\mathrm{i}}$ ranges smoothly from ${ }^{n} \mathbf{C}_{\mathrm{i}}$ to ${ }^{n+1} \overline{\mathbf{C}}$. Thus, there is no danger of "wild oscillations" of the solution, which are typical for explicit time stepping schemes dealing with large time steps. The algorithm is unconditionally stable since the solution remains finite for arbitrary time steps. Like the classical EBM, the algorithm is first order accurate (see Appendix B).

In case of a relaxation process with $\mathbf{C}=$ const, the Clausius-Duhem inequality (4) requires that the free energy is a decreasing function of time. Let us analyze the dissipation properties of the presented algorithm in case of stress relaxation. We consider a local relaxation process with $\mathbf{C}=$ const and $\mathbf{C}_{\mathrm{i}}={ }^{n} \mathbf{C}_{\mathrm{i}}$ as prescribed initial condition at $t_{n}$. In that case, it can be shown that $\psi\left(\mathbf{C}^{n+1} \mathbf{C}_{\mathrm{i}}^{-1}\right)$ is a monotonically decreasing function of $\Delta t$ (see Appendix $\mathrm{C}$ ). Thus, the relaxation property of the exact solution is qualitatively reproduced by the numerical solution.

Note that the evolution equations (10) are invariant under isochoric change of the reference configuration (see [56,59]). More precisely, let $\mathbf{F}_{0}$ be a constant tensor such that $\operatorname{det} \mathbf{F}_{0}=1$. The new reference configuration is introduced as $\widetilde{K}^{\text {new }}=\mathbf{F}_{0} \widetilde{K}$. Thus, the corresponding deformation gradient (relative deformation gradient) is then given by $\mathbf{F}^{\text {new }}=\mathbf{F F}_{0}^{-1}$. Along with the "old" quantities $\mathbf{C}$ and $\mathbf{C}_{\mathbf{i}}$, we consider their new counterparts

$$
\mathbf{C}^{\text {new }}=\mathbf{F}_{0}^{-\mathrm{T}} \mathbf{C F}_{0}^{-1}, \quad \mathbf{C}_{\mathrm{i}}^{\text {new }}=\mathbf{F}_{0}^{-\mathrm{T}} \mathbf{C}_{\mathrm{i}} \mathbf{F}_{0}^{-1} .
$$

In that case, it can be easily shown that the evolution of $\mathbf{C}_{\mathrm{i}}^{\text {new }}$ is governed by (10) if all "old" variables are formally replaced by their new counterparts. The same invariance requirement also can be formulated for the time-stepping procedure: For a fixed time step $\Delta t$, we denote the numerical solution by ${ }^{n+1} \mathbf{C}_{\mathrm{i}}=\mathfrak{N}\left({ }^{n+1} \mathbf{C},{ }^{n} \mathbf{C}_{\mathrm{i}}\right)$. The numerical scheme $\mathfrak{N}$ is called invariant under the reference change if

$$
\mathfrak{N}\left(\mathbf{F}_{0}^{-\mathrm{T}}{ }^{n+1} \mathbf{C ~ F}_{0}^{-1}, \mathbf{F}_{0}^{-\mathrm{T}}{ }^{n} \mathbf{C}_{\mathrm{i}} \mathbf{F}_{0}^{-1}\right)=\mathbf{F}_{0}^{-\mathrm{T}} \mathfrak{N}\left({ }^{n+1} \mathbf{C},{ }^{n} \mathbf{C}_{\mathrm{i}}\right) \mathbf{F}_{0}^{-1} .
$$

This property can be checked for the scheme (23). Indeed, since $\operatorname{det} \mathbf{F}_{0}=1$, we have

$$
\overline{\mathbf{F}_{0}^{-T_{n}} \mathbf{C}_{\mathrm{i}} \mathbf{F}_{0}^{-1}+\frac{\Delta t \mu}{\eta} \overline{\mathbf{F}_{0}^{-\mathrm{T}}{ }^{n+1} \mathbf{C} \mathbf{F}_{0}^{-1}}}=\mathbf{F}_{0}^{-\mathrm{T}} \overline{{ }^{n} \mathbf{C}_{\mathrm{i}}+\frac{\Delta t \mu}{\eta}{ }^{n+1} \overline{\mathbf{C}}} \mathbf{F}_{0}^{-1} .
$$


Remark 4 One possible application of the invariance property (24) is as follows. By choosing $\mathbf{F}_{0}:=\left({ }^{n} \mathbf{C}_{\mathrm{i}}\right)^{1 / 2}$ we get ${ }^{n} \mathbf{C}_{\mathrm{i}}^{\text {new }}=\mathbf{1}$. Thus, any algorithm computing $\mathfrak{N}\left({ }^{n+1} \mathbf{C}, \mathbf{1}\right)$ would be sufficient to restore the general scheme $\mathfrak{N}$. In other words, it is sufficient to perform time-stepping for zero initial conditions for the inelastic strain, which corresponds to ${ }^{n} \mathbf{C}_{\mathrm{i}}=\mathbf{1}$.

Within implicit, deformation driven finite element procedures, the consistent tangent operator is required for iterative solving for global balance of linear momentum [64]. An explicit expression for the consistent tangent is presented in Appendix D, and its symmetry is proved.

It can be shown that the numerical solution (23) corresponds to the exact solution of a relaxation problem with a fixed $\mathbf{C}$ at a certain time instance $t \approx t_{n+1}$. More precisely, consider the initial value problem $(10),(11)$ with $\mathbf{C}(t)=$ const. Then the exact solution

can be represented as $\mathbf{C}_{\mathrm{i}}(t)=\overline{\mathbf{C}_{\mathrm{i}}^{0}+\varphi(t) \overline{\mathbf{C}}}$ with $\varphi\left(t^{0}\right)=0$ and $\varphi(t) \approx \frac{\mu\left(t-t^{0}\right)}{\eta}$ as $t \rightarrow t^{0}$ (see Appendix E).

\subsection{Explicit update formula in Eulerian formulation}

In this subsection we rewrite the explicit update formula (23) in terms of tensors which operate on the current configuration. Toward that end, for each time interval $\left(t_{n}, t_{n+1}\right)$, we introduce the so-called trial elastic left Cauchy-Green tensor under the assumption of a frozen inelastic flow. Having in mind that $\hat{\mathbf{B}}_{\mathrm{e}}=\mathbf{F} \mathbf{C}_{\mathrm{i}}^{-1} \mathbf{F}^{\mathrm{T}}$, we get the trial elastic strain in the form

$$
{ }^{n+1} \hat{\mathbf{B}}_{\mathrm{e}}^{\text {trial }}={ }^{n+1} \mathbf{F}^{n} \mathbf{C}_{\mathrm{i}}^{-1}{ }^{n+1} \mathbf{F}^{\mathrm{T}} .
$$

Multiplying (23) with ${ }^{n+1} \overline{\mathbf{F}}^{-\mathrm{T}}$ from the left and with ${ }^{n+1} \overline{\mathbf{F}}^{-1}$ from the right, we get

$$
{ }^{n+1} \overline{\hat{\mathbf{B}}}_{\mathrm{e}}^{-1}=\overline{\left({ }^{n+1} \overline{\hat{\mathbf{B}}_{\mathrm{e}}^{\text {trial }}}\right)^{-1}+\frac{\Delta t \mu}{\eta} \mathbf{1}}
$$

Taking the inelastic incompressibility into account, one gets

$$
{ }^{n+1} \hat{\mathbf{B}}_{\mathrm{e}}^{-1}=\left(\operatorname{det}^{n+1} \mathbf{F}\right)^{-2 / 3} \overline{\left({ }^{n+1} \overline{\hat{\mathbf{B}}_{\mathrm{e}}^{\text {trial }}}\right)^{-1}+\frac{\Delta t \mu}{\eta} \mathbf{1}}
$$

This is an explicit update formula for the evolution equation (18). Note that, similar to the product formula consistent with the operator split considered by Simo [63], ${ }^{n+1} \hat{\mathbf{B}}_{\mathrm{e}}$ is co-axial with ${ }^{n+1} \hat{\mathbf{B}}_{\mathrm{e}}^{\text {trial }}$. Within a time step, the explicit update formula (25) predicts the same stress response as the formula (23). 


\subsection{Exponential mapping: Lagrangian and Eulerian formulations}

Alternatively to the explicit update formula considered in this paper, a well-known implicit method based on the exponential mapping can be adopted:

$$
{ }^{n+1} \mathbf{C}_{\mathrm{i}}=\exp \left[\frac{\Delta t \mu}{\eta}\left({ }^{n+1} \overline{\mathbf{C}}^{n+1} \mathbf{C}_{\mathrm{i}}^{-1}\right)^{\mathrm{D}}\right]{ }^{n} \mathbf{C}_{\mathrm{i}} .
$$

By some algebraic computations, it can be shown that the invariance relation (24) holds for this method as well. Next, a proof that the symmetry property is retained by the algorithm even in case of a more general material model is presented in [54]. Moreover, the incompressibility of the inelastic flow is exactly retained. Thus, for the corresponding numerical solution, ${ }^{n+1} \mathbf{C}_{\mathrm{i}} \in \mathbb{M}$.

Observe that the numerical scheme obtained using the operator split technique (cf. Simo [63]) can be derived directly from (26). Indeed, by inverting both sides of (26), we get

$$
{ }^{n+1} \mathbf{C}_{\mathrm{i}}^{-1}={ }^{n} \mathbf{C}_{\mathrm{i}}^{-1} \exp \left[-\frac{\Delta t \mu}{\eta}\left({ }^{n+1} \overline{\mathbf{C}}^{n+1} \mathbf{C}_{\mathrm{i}}^{-1}\right)^{\mathrm{D}}\right] .
$$

Since the inelastic incompressibility is exactly retained by $(26), \operatorname{det}\left({ }^{n+1} \hat{\mathbf{B}}_{\mathrm{e}}^{-1}\right)=\left(\operatorname{det}{ }^{n+1} \mathbf{F}\right)^{2}$. Therefore

$$
{ }^{n+1} \mathbf{F}^{-\mathrm{T}}\left({ }^{n+1} \overline{\mathbf{C}}^{n+1} \mathbf{C}_{\mathrm{i}}^{-1}\right)^{\mathrm{D}}{ }^{n+1} \mathbf{F}^{-\mathrm{T}}=\left({ }^{n+1} \overline{\mathbf{B}}_{\mathrm{e}}\right)^{\mathrm{D}} .
$$

Multiplying (27) with ${ }^{n+1} \mathbf{F}$ from the left and with ${ }^{n+1} \mathbf{F}^{\mathrm{T}}$ from the right, we get using (28)

$$
{ }^{n+1} \hat{\mathbf{B}}_{\mathrm{e}}={ }^{n+1} \hat{\mathbf{B}}_{\mathrm{e}}^{\text {trial }} \exp \left[-\frac{\Delta t \mu}{\eta}\left({ }^{n+1} \widehat{\mathbf{B}}_{\mathrm{e}}\right)^{\mathrm{D}}\right] .
$$

Therefore, ${ }^{n+1} \hat{\mathbf{B}}_{\mathrm{e}}$ commutes with ${ }^{n+1} \hat{\mathbf{B}}_{\mathrm{e}}^{\text {trial }}$. Thus, this equation can be rewritten in the form

$$
{ }^{n+1} \hat{\mathbf{B}}_{\mathrm{e}}=\exp \left[-\frac{\Delta t \mu}{\eta}\left({ }^{n+1} \hat{\mathbf{B}}_{\mathrm{e}}\right)^{\mathrm{D}}\right]{ }^{n+1} \hat{\mathbf{B}}_{\mathrm{e}}^{\text {trial }}
$$

The well-known implicit update formula is restored (see, for instance, equation (44) in [51]). We stress that the nonlinear equations (26) and (29) represent one and the same method, written in two different formulations. For a given time step, these equations are equivalent since they predict the same stress response. 


\section{Numerical results}

\subsection{Accuracy testing for a single Maxwell element}

In order to test the accuracy of the explicit update formula (23), we consider a local loading program in the time interval $t \in[0,300]$ (time is measured in seconds)

$$
\mathbf{F}(t)=\overline{\mathbf{F}^{\prime}(t)},
$$

where $\mathbf{F}^{\prime}(t)$ is a piecewise linear function of time $t$ such that $\mathbf{F}^{\prime}(0)=\mathbf{F}_{1}, \mathbf{F}^{\prime}(100)=\mathbf{F}_{2}$, $\mathbf{F}^{\prime}(200)=\mathbf{F}_{3}$, and $\mathbf{F}^{\prime}(300)=\mathbf{F}_{4}$ with

$$
\begin{gathered}
\mathbf{F}_{1}:=\mathbf{1}, \quad \mathbf{F}_{2}:=2 \mathbf{e}_{1} \otimes \mathbf{e}_{1}+\frac{1}{\sqrt{2}}\left(\mathbf{e}_{2} \otimes \mathbf{e}_{2}+\mathbf{e}_{3} \otimes \mathbf{e}_{3}\right), \\
\mathbf{F}_{3}:=\mathbf{1}+\mathbf{e}_{1} \otimes \mathbf{e}_{2}, \quad \mathbf{F}_{4}:=2 \mathbf{e}_{2} \otimes \mathbf{e}_{2}+\frac{1}{\sqrt{2}}\left(\mathbf{e}_{1} \otimes \mathbf{e}_{1}+\mathbf{e}_{3} \otimes \mathbf{e}_{3}\right) .
\end{gathered}
$$

More precisely, we put here

$$
\mathbf{F}^{\prime}(t):=\left\{\begin{array}{ll}
(1-t / 100) \mathbf{F}_{1}+(t / 100) \mathbf{F}_{2} & \text { if } t \in[0,100] \\
(2-t / 100) \mathbf{F}_{2}+(t / 100-1) \mathbf{F}_{3} & \text { if } t \in(100,200] \\
(3-t / 100) \mathbf{F}_{3}+(t / 100-2) \mathbf{F}_{4} & \text { if } t \in(200,300]
\end{array} .\right.
$$

The reference configuration is assumed to be stress free at $t=0$. Thus, we put $\left.\mathbf{C}_{\mathrm{i}}\right|_{t=0}=\mathbf{1}$. The following values of the material parameters are used: $\eta=400 \mathrm{MPa} \mathrm{s}, \mu=40 \mathrm{MPa}$. The numerical solution of the initial value problem obtained with extremely small time step $(\Delta t=0.001)$ will be referred to as the exact solution $\mathbf{C}_{\mathrm{i}}^{\text {exact }}$. The numerical solutions with $\Delta t=1$ and $\Delta t=0.5$ are denoted by $\mathbf{C}_{\mathrm{i}}^{\text {numer }}$. The error $\left\|\mathbf{C}_{\mathrm{i}}^{\text {numer }}-\mathbf{C}_{\mathrm{i}}^{\text {exact }}\right\|$ is plotted versus time in Fig. 2 for three different methods. The explicit update (23) is abbreviated as Euler-backward method with subsequent correction (EBMSC). Additionally to EBMSC, the classical EBM method and the exponential method (EM) are considered in this subsection. Since these three methods are first order accurate, the error is proportional to $\Delta t$. Moreover, in accordance with [56], there is no error accumulation in case of geometric integrators (EBMSC and EM). More precisely, the error is uniformly bounded by $C \Delta t$ where the constant $C$ does not depend on the size of the entire time interval [56]. Next, since the incompressibility condition is violated by EBM, the geometric property (12) is not preserved and the numerical error tends to accumulate over time. The geometric integrators EBMSC and EM are equivalent in terms of accuracy. The simulated stress response is presented in Fig. 3 for three different time steps $\Delta t$.

Next, the accuracy is tested for varying viscosity $\eta$ with a fixed $\mu=40 \mathrm{MPa}, \Delta t=10$ and loading programm given by (30). The largest relative error is observed for the smallest values of $\eta$, although the overall stress level tends to zero (see the top part of Fig. 4). Indeed, for smaller $\eta$ the problems becomes stiffer. Although the implicit schemes are 

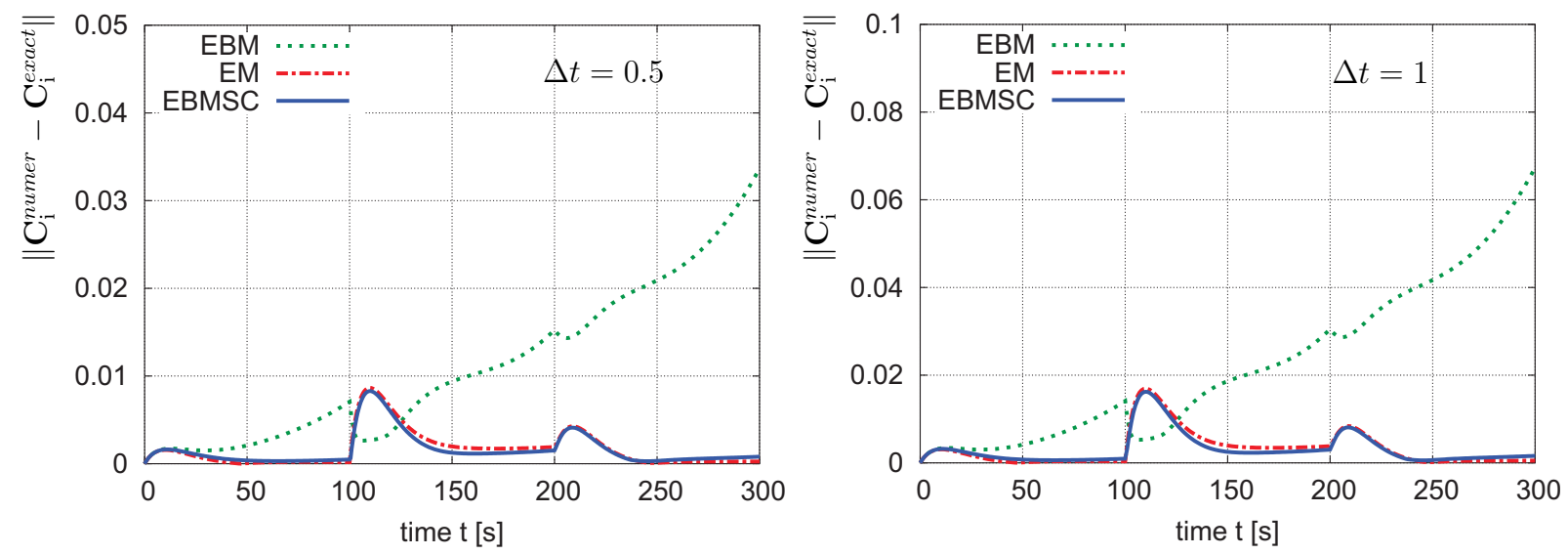

Fig. 2. Plots of the numerical error pertaining to three different algorithms: classical Euler backward (EBM), exponential method (EM), and the Euler backward with subsequent correction (EBMSC). Explicit update formula for EBMSC is given by (23). All methods are first order accurate. Geometric integrators (EBMSC and EM) prevent the error accumulation.

robust, smaller time steps are still required to reduce the numerical error. In case of large $\eta$, the inelastic flow is insignificant and the material response becomes nearly hyperelastic (see the bottom part of Fig. 4).

\subsection{Application to visoelasticity within the FEM}

\subsubsection{Material model of finite strain viscoelasticity}

In this section, a practical application of the proposed integration algorithm to the modeling of viscoelastic material response is discussed. As a typical example, a constitutive model proposed by Reese and Govindjee $[51,10]$ is considered. The rheological interpretation of the model consists of a single hyperelastic spring (Hooke body) for the representation of equilibrium stresses and $N$ Maxwell bodies which are connected in parallel to the Hooke body to represent viscous effects, see Fig. 1b. Each of these Maxwell bodies is governed by equations presented in Section 2. The total free energy is given as a sum of isotropic functions as follows (cf. $[51,10])$ :

$$
\psi=\psi_{\mathrm{eq}}(\mathbf{C})+\sum_{m=1}^{N} \psi_{\mathrm{ov}, m}\left(\mathbf{C C}_{\mathrm{i}, m}^{-1}\right),
$$

where the equilibrium part is modeled by the hyperelastic Yeoh model [68] in combination with an additional volumetric part

$$
\rho_{\mathrm{R}} \psi_{\mathrm{eq}}(\mathbf{C})=\sum_{n=1}^{3} c_{i 0}(\operatorname{tr} \overline{\mathbf{C}}-3)^{n}+\frac{9 k}{2}\left((\operatorname{det} \mathbf{F})^{1 / 3}-1\right)^{2} .
$$

Here, $c_{10}, c_{20}$, and $c_{30}$ are material parameters of the Yeoh material; $k$ stands for the bulk modulus. Observe that the volumetric stress response depends on the volume ratio, given 

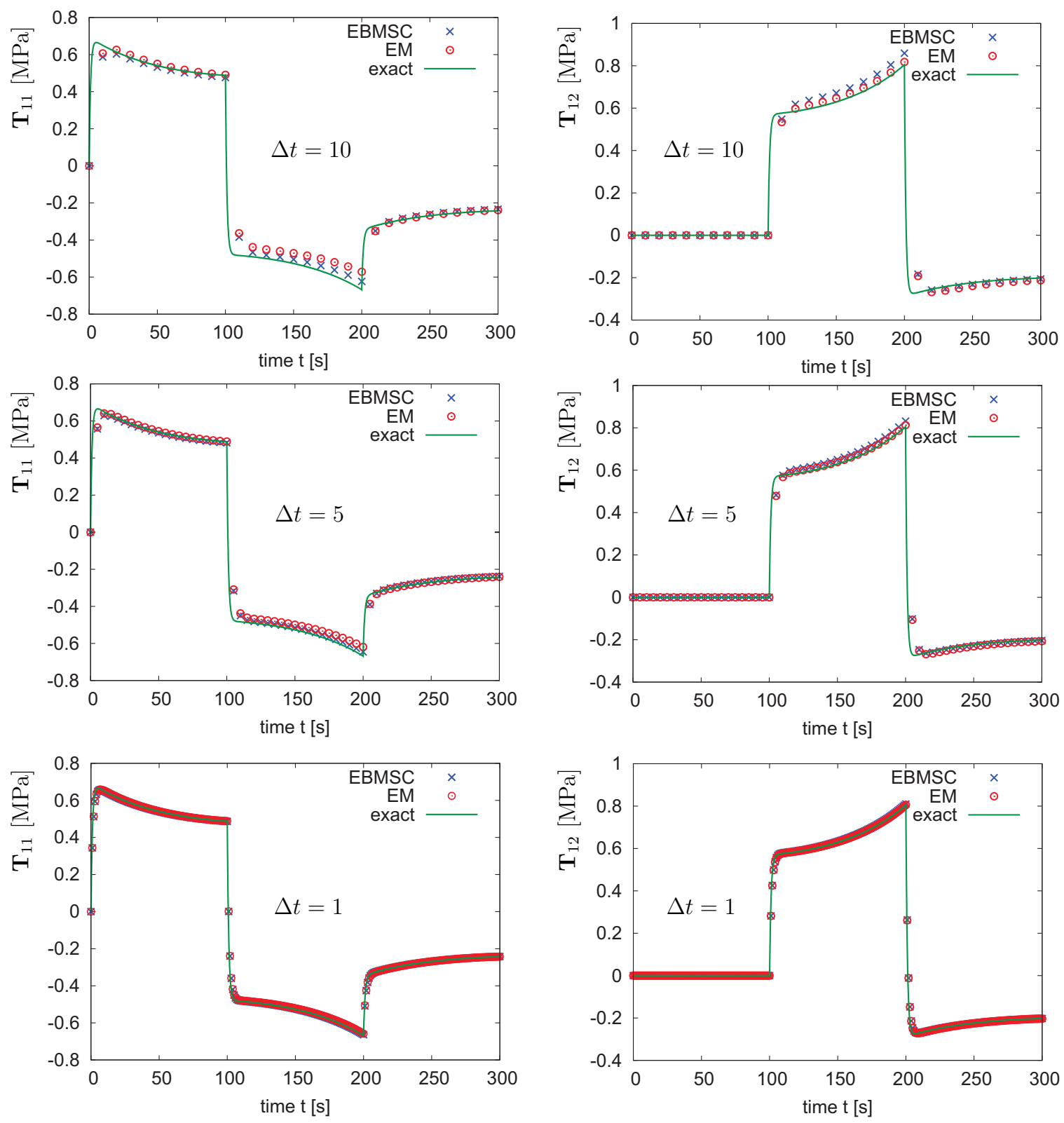

Fig. 3. Simulated stress response in terms of Cauchy stresses for $\Delta t=10$ (top), $\Delta t=5$ (middle), and $\Delta t=1$ (bottom). Since both methods are first order accurate, the error is proportional to $\Delta t$.

by $\operatorname{det} \mathbf{F} 8$ Next, the free energy for the $m^{\text {th }}$ Maxwell body reads as

$$
\rho_{\mathrm{R}} \psi_{\mathrm{ov}, m}=\rho_{\mathrm{R}} \psi_{\mathrm{ov}, m}\left(\mathbf{C C}_{\mathrm{i}, k}^{-1}\right)=\frac{\mu_{m}}{2}\left(\operatorname{tr} \overline{\mathbf{C C}_{\mathrm{i}, m}^{-1}}-3\right), \quad m=1,2, \ldots, N
$$

Here, $\mu_{m} \geq 0$ is the shear modulus of the $m^{\text {th }}$ element and $\mathbf{C}_{\mathbf{i}, m}$ is the corresponding inelastic tensor of right Cauchy Green type. The evolution of each of these variables is

$\overline{8}$ The volumetric ansatz presented in (31) is implemented in MSC.MARC [42]. Obviously, any alternative ansatz for the volumetric part can be used as well. 

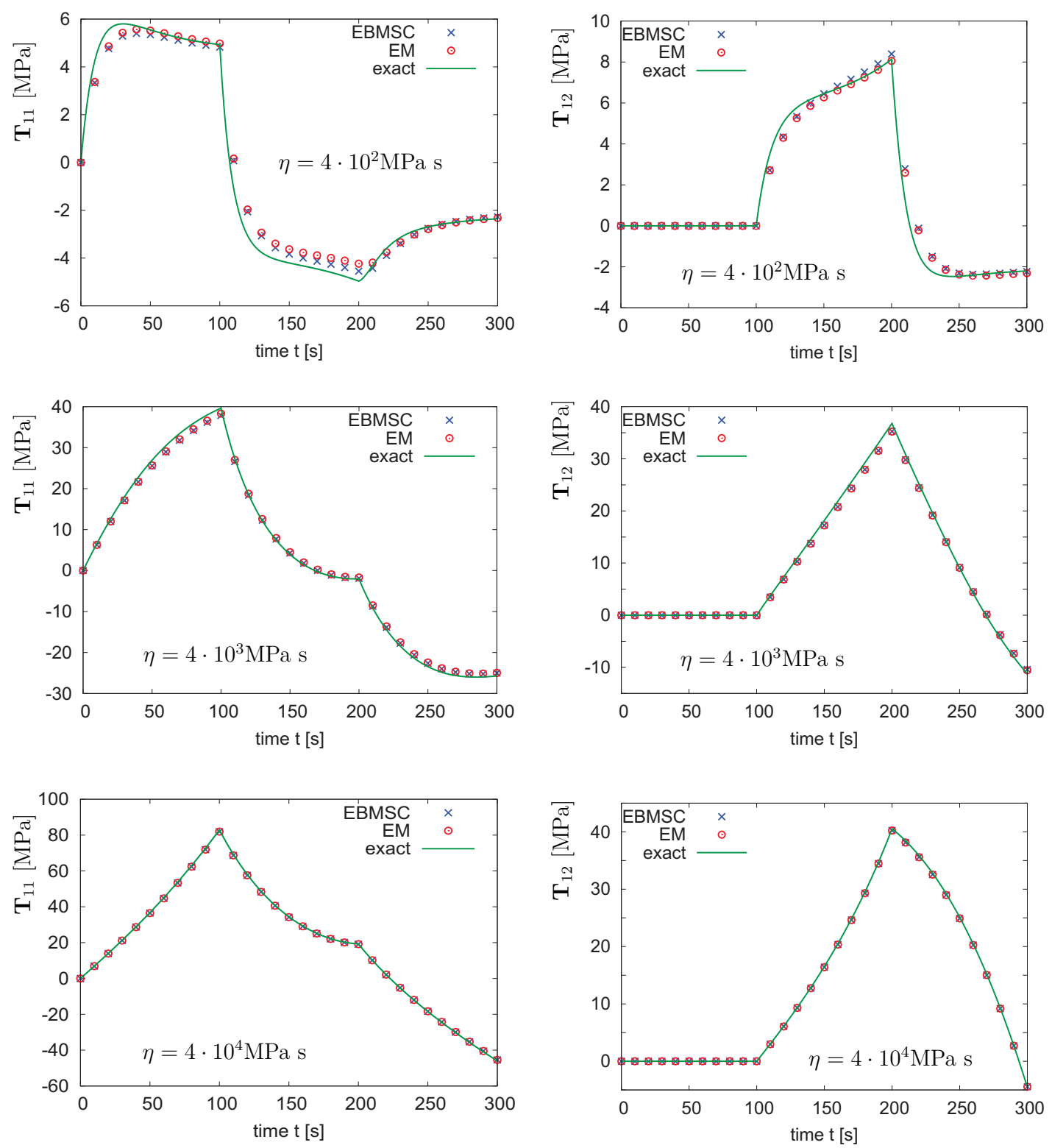

Fig. 4. Simulated stress response in terms of Cauchy stresses for different values of $\eta$ : $\eta=4 \cdot 10^{2} \mathrm{MPa} \mathrm{s}$ (top), $\eta=4 \cdot 10^{3} \mathrm{MPa} \mathrm{s}$ (middle), and $\eta=4 \cdot 10^{4} \mathrm{MPa} \mathrm{s}$ (bottom). The relative error is higher for stiffer problems with small $\eta$.

governed by equations of type (10). The resulting system of constitutive equations is as follows:

$$
\begin{gathered}
\tilde{\mathbf{T}}=\tilde{\mathbf{T}}_{\mathrm{eq}}+\sum_{m=1}^{N} \tilde{\mathbf{T}}_{\mathrm{ov}, m}, \\
\tilde{\mathbf{T}}_{\mathrm{eq}}=\left(2 c_{10}+4 c_{20}(\operatorname{tr} \overline{\mathbf{C}}-3)+6 c_{30}(\operatorname{tr} \overline{\mathbf{C}}-3)^{2}\right) \overline{\mathbf{C}}^{\mathrm{D}} \mathbf{C}^{-1}+3 k(\operatorname{det} \mathbf{F})^{1 / 3}\left((\operatorname{det} \mathbf{F})^{1 / 3}-1\right) \mathbf{C}^{-1}, \\
\tilde{\mathbf{T}}_{\mathrm{ov}, m}=\mu_{m} \mathbf{C}^{-1}\left(\overline{\mathbf{C}} \mathbf{C}_{\mathrm{i}, m}^{-1}\right)^{\mathrm{D}}, \quad \dot{\mathbf{C}}_{\mathrm{i}, m}=\frac{\mu_{m}}{\eta_{m}}\left(\overline{\mathbf{C}}_{\mathrm{i}, m}^{-1}\right)^{\mathrm{D}} \mathbf{C}_{\mathrm{i}, m}, \quad m=1,2, \ldots, N,
\end{gathered}
$$

where $\eta_{m} \geq 0$ denotes the viscosity of the $m^{\text {th }}$ Maxwell body. 

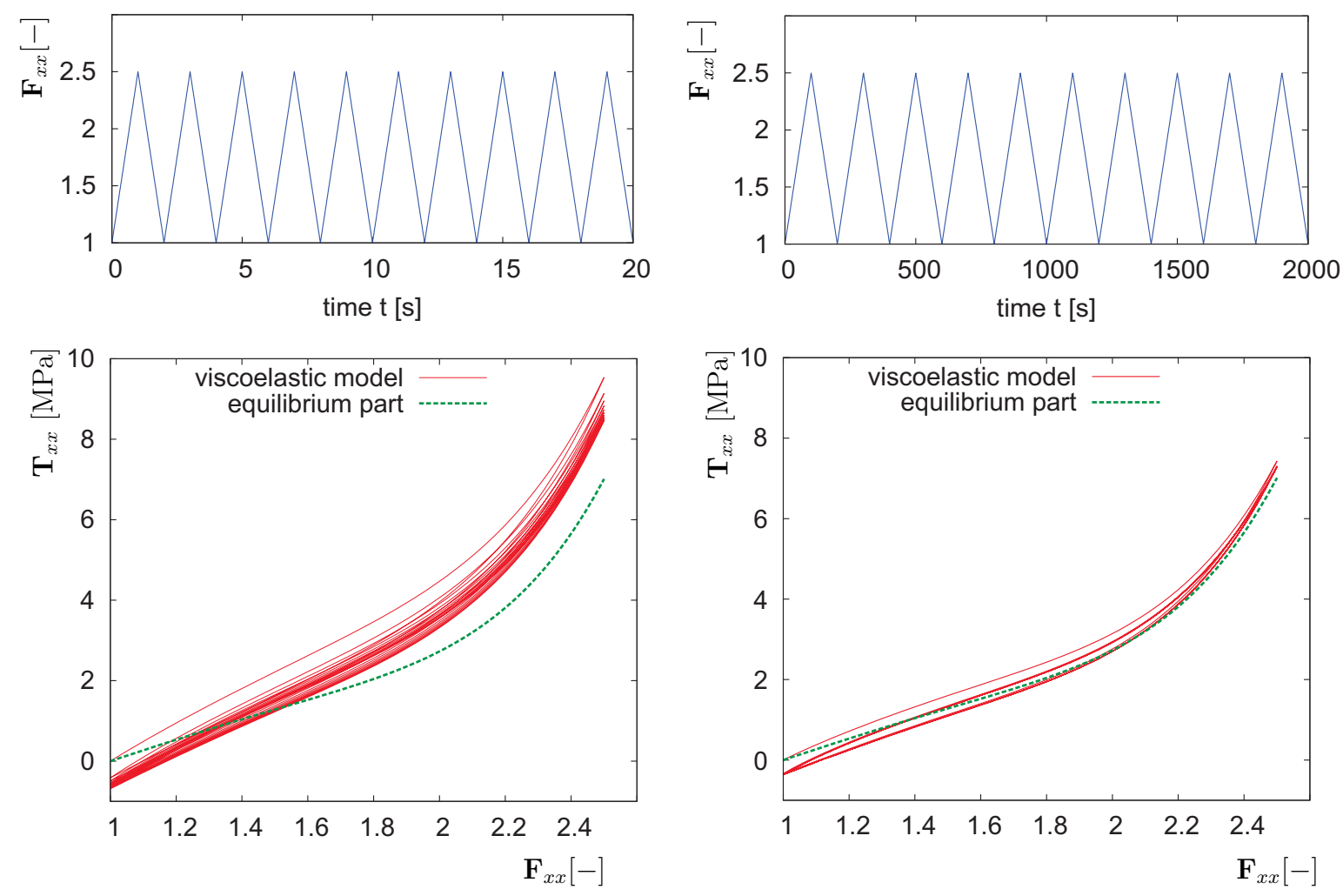

Fig. 5. Simulated stress response under uniaxial cyclic loading for different strain rates: for $\left|\dot{\mathbf{F}}_{x x}\right|=1.5 \mathrm{~s}^{-1}$ (left) and for $\left|\dot{\mathbf{F}}_{x x}\right|=0.015 \mathrm{~s}^{-1}$ (right).

The material model is implemented into commercial finite element software MSC.MARC ${ }^{\circledR}$, making use of the user subroutine HYPELA2. Since the total strain is prescribed at each point of Gauss integration, the numerical treatment of each of the Maxwell elements is fully independent from other Maxwell elements. For each of them, the corresponding evolution equations are solved with the help of the explicit update formula (23) and the consistent tangent operator is computed explicitly (cf. Appendix D). In order to avoid volume locking effects, a mixed u-p-formulation is adopted [65]. For the subsequent simulations, a system of 4 Maxwell bodies is implemented with the material parameters from Tab. 1.

Table 1

Material parameters of a viscoelastic material $(m=1,2,3,4)$.

\begin{tabular}{|c|c|c|c|c|c|c|}
\hline$c_{10}[\mathrm{MPa}]$ & $c_{20}[\mathrm{MPa}]$ & $c_{30}[\mathrm{MPa}]$ & $k[\mathrm{MPa}]$ & $N[-]$ & $\mu_{m}[\mathrm{MPa}]$ & $\eta_{m}[\mathrm{MPa} \mathrm{s}]$ \\
\hline 0.45 & -0.048 & 0.011 & 1000 & 4 & 0.2 & $2 \cdot 10^{m-3}$ \\
\hline
\end{tabular}

First, in order to illustrate the stress response of the model, a uniaxial cyclic loading is simulated. The loading axis is fixed and coincides with the $x$-axis. In Fig. 5 the stress response under a strain driven loading is shown for two different loading rates. It can be seen that for small loading rates the stress response converges to the equilibrium path. Next, the simulated stress response for uniaxial relaxation test is represented in Fig. 6. Note that if the instant stress lies under the equilibrium stress, the relaxation process 

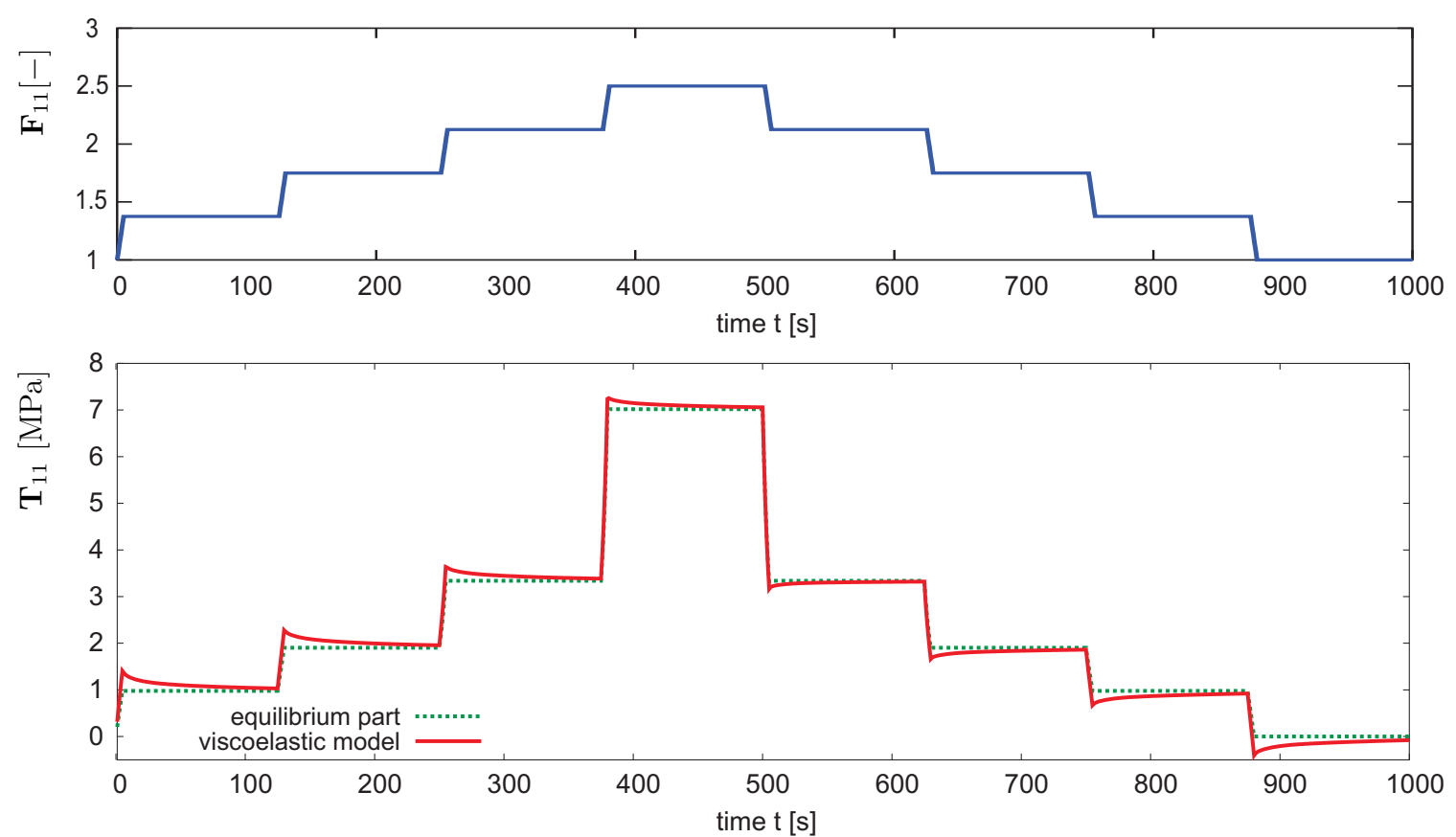

Fig. 6. Simulation results for uniaxial relaxation tests: loading program (top) and stress response (bottom).

results in increasing stresses.

\subsubsection{FEM solution of a representative boundary value problem}

A representative boundary value problem is solved in this subsection in order to test the stability properties of the proposed integration algorithm. Toward that end, the material model from the previous subsection is adopted to simulate the so-called rotary dynamics experiment used for the analysis of rubber materials. The corresponding experimental setup was originally proposed in [9] and applied later for the life-time prediction of rubber materials in $[28,29]$. Within this experiment, a shear loading with rotating axes is applied to two cylindrical specimens as shown in Fig. 79

The used finite element model is adopted from [21]. It represents a single cylinder of height $h=40 \mathrm{~mm}$ and of diameter $d=10 \mathrm{~mm}$ (Fig. 8). Eight-node isoparametric threedimensional brick elements with trilinear interpolation and one extra node with a single degree of freedom for pressure (MSC.MARC element type 84) are used. The mesh consists of 7000 elements. Loads are applied only on the upper and lower surfaces in the following manner. Both surfaces are rigid and can rotate independently about corresponding rotation axes. Each rotation axis goes through the center of the corresponding surface in the normal direction. For the lower surface, the rotation axis is fixed and the rotation is free. For the upper surface, an initial displacement of the surface is submitted in $y$-direction as shown in Fig. 8, such that a simple shear loading is applied to the specimen. In the

$\overline{9}$ The animated version of the experiment can be seen at http://youtu.be/eNgjGE7upYY. 


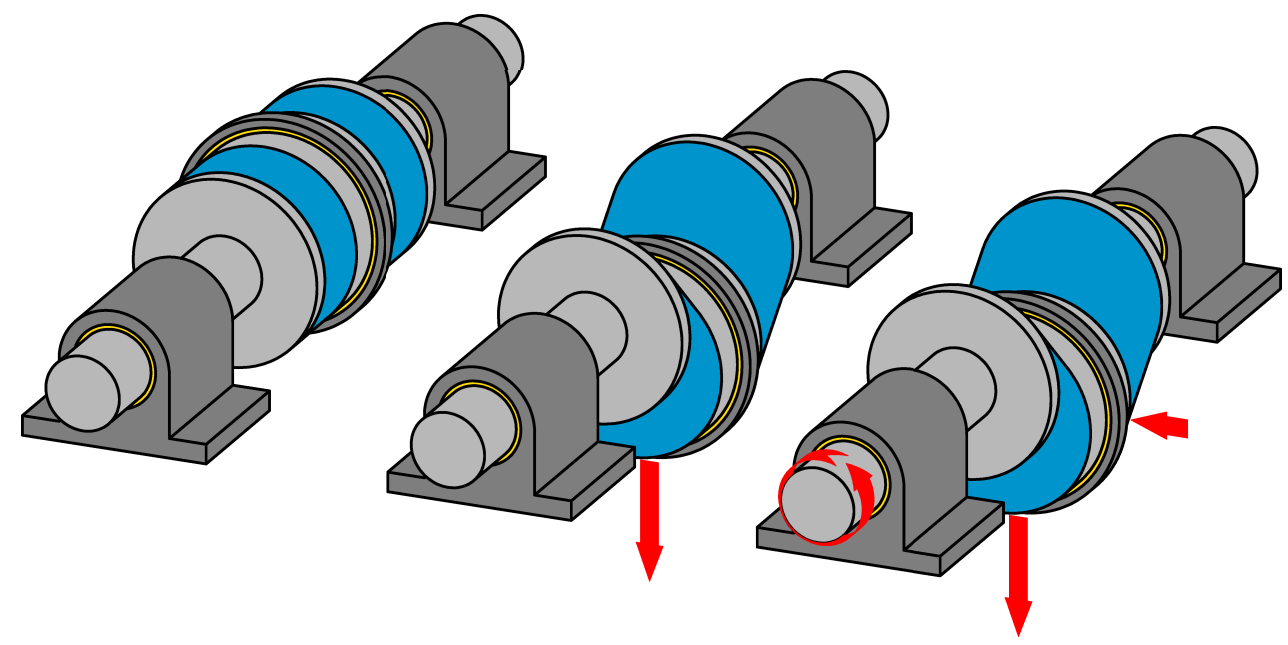

Fig. 7. Experimental setup for simple shear loading with rotating axes [28]: initial state (left), monotonic shear loading (middle), shear loading with rotating axes (right).

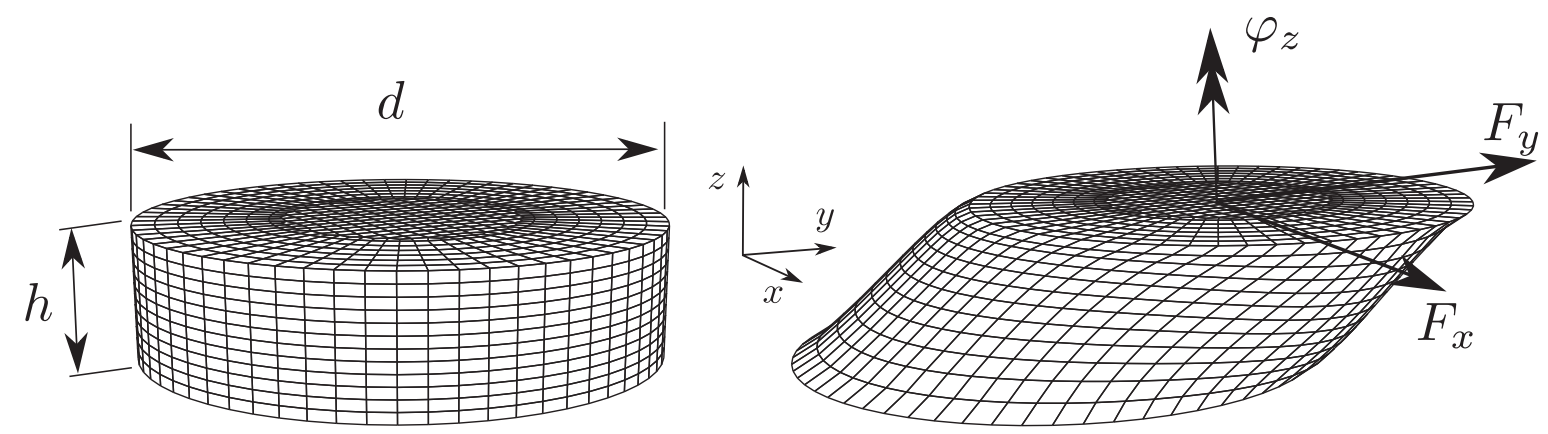

Fig. 8. FEM model of the rotary dynamics testing: initial configuration (left) and deformed configuration (right).

next loading step, the corresponding axis is fixed and a rotation of the upper surface is prescribed with a constant angular velocity $\dot{\varphi}_{z}=0.2 \pi / \mathrm{s}$. Thus, the sample is subjected to a non-proportional cyclic loading. Finally, after $300 \mathrm{~s}$, the rotation is stopped.

The numerical simulation was performed with the time step $\Delta t=0.25 \mathrm{~s}$. The material parameters of the viscoelastic material are taken from Tab. 1. No convergence difficulties were observed during the simulation whatsoever. The simulated reaction forces $F_{x}$ and $F_{y}$ are shown in Fig. 9. After approximately 10 revolutions of the sample, nearly stationary solution is observed. Finally, during the relaxation step, the reaction force $F_{x}$ disappears and the force $F_{y}$ tends to a certain equilibrium value.

\section{Discussion and conclusion}

A closed from solution for fully implicit integration algorithm is proposed. The algorithm corresponds to the classical Euler-backward method with a subsequent correction to enforce the incompressibility of the inelastic flow. The scheme is highly efficient, since no 

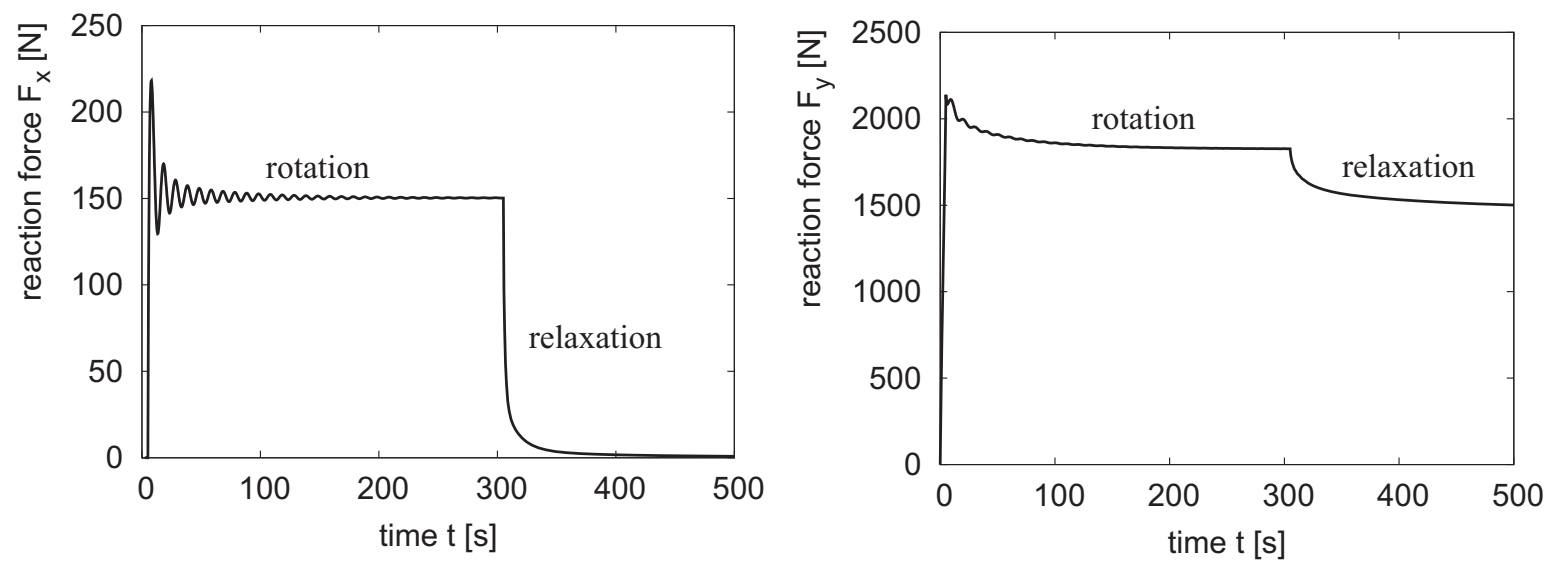

Fig. 9. Simulation results for reaction forces. FEM simulation performed in MSC.MARC using explicit update formula (23).

local iteration procedure is required. The numerical solution is well defined even for large time steps $\Delta t$. The resulting integration algorithm is unconditionally stable and first order accurate. The algorithm shows a similar accuracy as the well-known exponential scheme (EM). The consistent tangent operator is symmetric. The following properties of the exact solution are retained by the algorithm (23):

i symmetry and incompressibility: ${ }^{n+1} \mathbf{C}_{\mathrm{i}} \in \operatorname{Sym}$, $\operatorname{det}\left({ }^{n+1} \mathbf{C}_{\mathrm{i}}\right)=1$,

ii positive definiteness: ${ }^{n+1} \mathbf{C}_{\mathrm{i}}>0$,

iii the solution ${ }^{n+1} \mathbf{C}_{\mathrm{i}}$ is a smooth function of $\Delta t$,

iv for the stress relaxation with $\mathbf{C}=$ const, the free energy $\psi\left(\mathbf{C}^{n+1} \mathbf{C}_{\mathrm{i}}{ }^{-1}\right)$ is a monotonically decreasing function of $\Delta t$,

$\mathrm{v}$ numerical solution remains invariant under the isochoric change of the reference configuration.

No error accumulation is observed due to the exact preservation of the incompressibility constraint, in accordance to the theoretical results from [56].

The explicit update formula is derived for a special variant of the finite strain Maxwell fluid which is widely adopted in material modelling. Some modifications of the algorithm are possible to cover more general material behavior. In particular, the case of processdependent viscosity can be considered. Moreover, the Neo-Hookean type of hyperelasticity can be replaced by more general constitutive assumptions. Application of the method to elasto-plasticity with different types of nonlinear hardening seems promising.

\section{Acknowledgement}

This research was supported by the German National Science Foundation (DFG) within SFB 692, PAK 273 and SFB/TR 39. 


\section{Appendix A (explicit update formula for EBM)}

Starting from (22), we derive an explicit update formula for the classical Euler-backward method. It is sufficient to obtain a closed-form relation for $\beta$ which appears in (22). First, we abbreviate:

$$
\boldsymbol{\Phi}:={ }^{n} \mathbf{C}_{\mathrm{i}}+\frac{\mu \Delta t}{\eta}{ }^{n+1} \overline{\mathbf{C}} .
$$

By computing the inverse of both sides of (22), we get

$$
\left({ }^{n+1} \mathbf{C}_{\mathrm{i}}^{\mathrm{EBM}}\right)^{-1}=(1+\beta) \boldsymbol{\Phi}^{-1} .
$$

Substituting this result into the definition (21), we obtain a linear equation with respect to $\beta$

$$
\beta=\frac{1}{3} \frac{\mu \Delta t}{\eta} \operatorname{tr}\left((1+\beta)^{n+1} \mathbf{C} \boldsymbol{\Phi}^{-1}\right)
$$

After resolving it, we get

$$
\beta=\frac{\frac{1}{3} \frac{\mu \Delta t}{\eta} \operatorname{tr}\left({ }^{n+1} \mathbf{C} \boldsymbol{\Phi}^{-1}\right)}{1-\frac{1}{3} \frac{\mu \Delta t}{\eta} \operatorname{tr}\left({ }^{n+1} \mathbf{C} \boldsymbol{\Phi}^{-1}\right)} .
$$

Finally, substituting this into (22), the EBM solution is given by

$$
{ }^{n+1} \mathbf{C}_{\mathrm{i}}^{\mathrm{EBM}}=\left(1-\frac{1}{3} \frac{\mu \Delta t}{\eta} \operatorname{tr}\left({ }^{n+1} \mathbf{C} \boldsymbol{\Phi}^{-1}\right)\right) \boldsymbol{\Phi} .
$$

\section{Appendix B (convergence rate)}

Let us show that the method (19) is first order accurate. Toward that end we consider a time interval $\left(t_{n}, t_{n+1}\right)$. Let $\mathbf{C}_{\mathbf{i}}\left(t_{n+1}\right)$ be the exact solution to the problem (10) with the initial condition $\left.\mathbf{C}_{\mathrm{i}}\right|_{t=t_{n}}={ }^{n} \mathbf{C}_{\mathrm{i}}$. It is sufficient to show that there exists $C<\infty$ such that

$$
\left\|^{n+1} \mathbf{C}_{\mathrm{i}}-\mathbf{C}_{\mathrm{i}}\left(t_{n+1}\right)\right\| \leq C(\Delta t)^{2} \text { as } \Delta t \rightarrow 0 .
$$

It is well known that the EBM is first order accurate. Moreover, there exists $C_{1}<\infty$ such that

$$
\left\|^{n+1} \mathbf{C}_{\mathrm{i}}^{\mathrm{EBM}}-\mathbf{C}_{\mathrm{i}}\left(t_{n+1}\right)\right\| \leq C_{1}(\Delta t)^{2} \text { as } \Delta t \rightarrow 0 .
$$

On the other hand, $(\operatorname{det}(\mathbf{X}))^{-1 / 3}$ is a smooth function of $\mathbf{X}$ in vicinity of the exact solution $\mathbf{C}_{\mathrm{i}}\left(t_{n+1}\right)$. Therefore, for sufficiently small time steps, there exists a constant $C_{3}<\infty$ such that

$$
\begin{array}{r}
\mid\left(\operatorname{det}\left({ }^{n+1} \mathbf{C}_{\mathrm{i}}^{\mathrm{EBM}}\right)^{-1 / 3}-1|=|\left(\operatorname{det}\left({ }^{n+1} \mathbf{C}_{\mathrm{i}}^{\mathrm{EBM}}\right)^{-1 / 3}-\operatorname{det}\left(\mathbf{C}_{\mathrm{i}}\left(t_{n+1}\right)\right)^{-1 / 3} \mid \leq\right.\right. \\
C_{3}\left\|^{n+1} \mathbf{C}_{\mathrm{i}}^{\mathrm{EBM}}-\mathbf{C}_{\mathrm{i}}\left(t_{n+1}\right)\right\| \leq C_{1} C_{3}(\Delta t)^{2} \quad \text { as } \Delta t \rightarrow 0 .
\end{array}
$$


Thus, for small $\Delta t$, there exists $C_{4}<\infty$ such that

$$
\left\|\overline{{ }^{n+1} \mathbf{C}_{\mathrm{i}}^{\mathrm{EBM}}}-{ }^{n+1} \mathbf{C}_{\mathrm{i}}^{\mathrm{EBM}}\right\|=\mid\left(\operatorname{det}\left({ }^{n+1} \mathbf{C}_{\mathrm{i}}^{\mathrm{EBM}}\right)^{-1 / 3}-1 \mid\left\|^{n+1} \mathbf{C}_{\mathrm{i}}^{\mathrm{EBM}}\right\| \leq C_{4}(\Delta t)^{2} .\right.
$$

Finally, using the triangle inequality, we get the required estimation

$$
\begin{aligned}
\left\|^{n+1} \mathbf{C}_{\mathrm{i}}-\mathbf{C}_{\mathrm{i}}\left(t_{n+1}\right)\right\|=\left\|^{\overline{n+1} \mathbf{C}_{\mathrm{i}}^{\mathrm{EBM}}}-\mathbf{C}_{\mathrm{i}}\left(t_{n+1}\right)\right\| \leq \\
\left\|\overline{{ }^{n+1} \mathbf{C}_{\mathrm{i}}^{\mathrm{EBM}}}-{ }^{n+1} \mathbf{C}_{\mathrm{i}}^{\mathrm{EBM}}\right\|+\left\|^{n+1} \mathbf{C}_{\mathrm{i}}^{\mathrm{EBM}}-\mathbf{C}_{\mathrm{i}}\left(t_{n+1}\right)\right\| \leq\left(C_{4}+C_{1}\right)(\Delta t)^{2} .
\end{aligned}
$$

\section{Appendix $\mathrm{C}$ (energy release during relaxation)}

Let us show that $\psi\left(\mathbf{C}^{n+1} \mathbf{C}_{\mathrm{i}}^{-1}\right)$ is a monotonically decreasing function of $\Delta t$ for a fixed $\mathbf{C}$, where ${ }^{n+1} \mathbf{C}_{\mathrm{i}}$ is given by the explicit update formula (23). We make use of the fact that the free energy $\psi\left(\mathbf{C} \mathbf{C}_{\mathrm{i}}{ }^{-1}\right)$ is invariant under the isochoric change of the reference

configuration. Thus, due to the invariance of the integration algorithm with respect to the reference change, we may assume that $\overline{\mathbf{C}}=\mathbf{1}$. In that case, the update formula takes the simple form

$$
{ }^{n+1} \mathbf{C}_{\mathrm{i}}=\overline{\boldsymbol{\Phi}}, \quad \boldsymbol{\Phi}:={ }^{n} \mathbf{C}_{\mathrm{i}}+\frac{\mu \Delta t}{\eta} \mathbf{1}
$$

For $\overline{\mathbf{C}}=\mathbf{1}$, it follows from (8) that

$$
\frac{d}{d \Delta t} \psi\left(\mathbf{C}^{n+1} \mathbf{C}_{\mathrm{i}}^{-1}\right)=\frac{\mu}{2 \rho_{\mathrm{R}}} \frac{d}{d \Delta t}\left(\operatorname{tr}\left(\mathbf{C}_{\mathrm{i}}^{-1}\right)\right)=-\frac{\mu}{2 \rho_{\mathrm{R}}} \operatorname{tr}\left({ }^{n+1} \mathbf{C}_{\mathrm{i}}^{-1} \frac{d}{d \Delta t}\left({ }^{n+1} \mathbf{C}_{\mathrm{i}}\right){ }^{n+1} \mathbf{C}_{\mathrm{i}}^{-1}\right)
$$

Note that

$$
\frac{d}{d \Delta t} \boldsymbol{\Phi}=\frac{\mu}{\eta} \mathbf{1}, \quad \frac{d}{d \Delta t}(\operatorname{det} \boldsymbol{\Phi})^{-1 / 3}=-\frac{1}{3} \frac{\mu}{\eta}(\operatorname{det} \boldsymbol{\Phi})^{-1 / 3} \operatorname{tr}\left(\boldsymbol{\Phi}^{-1}\right) .
$$

Thus, differentiating $(32)_{1}$ and using (34), we get

$$
\frac{d}{d \Delta t}{ }^{n+1} \mathbf{C}_{\mathrm{i}}=(\operatorname{det} \boldsymbol{\Phi})^{-1 / 3} \frac{\mu}{\eta}\left[\mathbf{1}-\frac{1}{3} \operatorname{tr}\left(\boldsymbol{\Phi}^{-1}\right) \boldsymbol{\Phi}\right]
$$

Substituting this result into (33) and taking into account that ${ }^{n+1} \mathbf{C}_{\mathrm{i}}^{-1}=(\operatorname{det} \boldsymbol{\Phi})^{1 / 3} \boldsymbol{\Phi}^{-1}$, we get

$$
\begin{aligned}
& \frac{d}{d \Delta t} \psi\left(\mathbf{C}^{n+1} \mathbf{C}_{\mathrm{i}}^{-1}\right)=-(\operatorname{det} \boldsymbol{\Phi})^{1 / 3} \frac{\mu^{2}}{2 \rho_{\mathrm{R}} \eta} \operatorname{tr}\left(\boldsymbol{\Phi}^{-1}\left[\mathbf{1}-\frac{1}{3} \operatorname{tr}\left(\boldsymbol{\Phi}^{-1}\right) \boldsymbol{\Phi}\right] \boldsymbol{\Phi}^{-1}\right)= \\
&-(\operatorname{det} \boldsymbol{\Phi})^{1 / 3} \frac{\mu^{2}}{2 \rho_{\mathrm{R}} \eta} \operatorname{tr}\left(\boldsymbol{\Phi}^{-1}\left(\boldsymbol{\Phi}^{-1}\right)^{\mathrm{D}}\right)=-(\operatorname{det} \boldsymbol{\Phi})^{1 / 3} \frac{\mu^{2}}{2 \rho_{\mathrm{R}} \eta}\left\|\left(\boldsymbol{\Phi}^{-1}\right)^{\mathrm{D}}\right\|^{2} \leq 0 .
\end{aligned}
$$

In particular, the free energy of the numerical solution for relaxation processes is a decreasing function of $\Delta t$. 


\section{Appendix D (consistent tangent operator)}

Combining (9) and (23), we define a function $\tilde{\mathbf{T}}_{1}(\mathbf{C})$ through

$$
\tilde{\mathbf{T}}_{1}(\mathbf{C})=\mu \mathbf{C}^{-1}\left(\overline{\mathbf{C}}\left(\overline{{ }^{n} \mathbf{C}_{\mathrm{i}}+\frac{\mu \Delta t}{\eta} \overline{\mathbf{C}}}\right)^{-1}\right)^{\mathrm{D}} .
$$

Next, we abbreviate

$$
\boldsymbol{\Phi}:={ }^{n} \mathbf{C}_{\mathrm{i}}+\frac{\mu \Delta t}{\eta} \overline{\mathbf{C}}, \quad \mathbf{C}_{\mathrm{i}}(\mathbf{C}): \overline{{ }^{n} \mathbf{C}_{\mathrm{i}}+\frac{\mu \Delta t}{\eta} \overline{\mathbf{C}}}=\overline{\boldsymbol{\Phi}} .
$$

Using notations from [55], the consistent tangent operator is explicitly given by

$$
\begin{gathered}
\frac{\partial \tilde{\mathbf{T}}_{1}(\mathbf{C})}{\partial \mathbf{C}} \stackrel{(35)}{=} \frac{\partial\left(\mu \mathbf{C}^{-1}\left(\overline{\mathbf{C}} \mathbf{C}_{\mathrm{i}}^{-1}\right)^{\mathrm{D}}\right)}{\partial \mathbf{C}}=-\mu \mathbf{C}^{-1} \odot\left(\mathbf{C}^{-1}\left(\overline{\mathbf{C}} \mathbf{C}_{\mathrm{i}}^{-1}\right)^{\mathrm{D}}\right)+ \\
\mu(\operatorname{det} \mathbf{C})^{-1 / 3} \mathbf{C}^{-1} \cdot\left\{-\frac{1}{3}\left(\mathbf{C C}_{\mathrm{i}}^{-1}\right)^{\mathrm{D}} \otimes \mathbf{C}^{-1}+\mathbb{P}:\left(\mathbb{I} \cdot \mathbf{C}_{\mathrm{i}}^{-1}+\mathbf{C} \cdot \frac{\partial \mathbf{C}_{\mathrm{i}}^{-1}}{\partial \mathbf{C}}\right)\right\}, \\
\frac{\partial \mathbf{C}_{\mathrm{i}}^{-1}}{\partial \mathbf{C}}=-\left(\mathbf{C}_{\mathrm{i}}^{-1} \odot \mathbf{C}_{\mathrm{i}}^{-1}\right): \frac{\partial \mathbf{C}_{\mathrm{i}}}{\partial \mathbf{C}}, \\
\frac{\partial \mathbf{C}_{\mathrm{i}}}{\partial \mathbf{C}} \stackrel{(36)_{2}}{=}(\operatorname{det} \boldsymbol{\Phi})^{-1 / 3}\left(\mathbb{I}-\frac{1}{3}\left(\mathbf{\Phi} \otimes \mathbf{\Phi}^{-1}\right)\right): \frac{\partial \mathbf{\Phi}}{\partial \mathbf{C}}, \\
\frac{\partial \mathbf{\Phi}}{\partial \mathbf{C}} \stackrel{(36)_{1}}{=} \frac{\mu \Delta t}{\eta}(\operatorname{det} \mathbf{C})^{-1 / 3}\left(\mathbb{I}-\frac{1}{3}\left(\mathbf{C} \otimes \mathbf{C}^{-1}\right)\right) .
\end{gathered}
$$

Now let us prove the symmetry of the tangent operator $\frac{\partial \tilde{\mathbf{T}}_{1}(\mathbf{C})}{\partial \mathbf{C}}$. First, note that the material tangent resulting for frozen inelastic flow $\left(\mathbf{C}_{\mathrm{i}}=\right.$ const $)$ corresponds to the symmetric tangent operator of a hyperelastic material:

$$
\left.\frac{\partial\left(\mu \mathbf{C}^{-1}\left(\overline{\mathbf{C}} \mathbf{C}_{\mathrm{i}}^{-1}\right)^{\mathrm{D}}\right)}{\partial \mathbf{C}}\right|_{\mathbf{C}_{\mathrm{i}}=\text { const }} \in \text { Sym. }
$$

Thus, the symmetry of $\frac{\partial \tilde{\mathbf{T}}_{1}(\mathbf{C})}{\partial \mathbf{C}}$ is equivalent to the symmetry of $\mathbb{T}$ defined through

$$
\begin{aligned}
& \mathbb{T}:=\frac{\partial \tilde{\mathbf{T}}_{1}(\mathbf{C})}{\partial \mathbf{C}}-\left.\frac{\partial\left(\mu \mathbf{C}^{-1}\left(\overline{\mathbf{C}} \mathbf{C}_{\mathrm{i}}^{-1}\right)^{\mathrm{D}}\right)}{\partial \mathbf{C}}\right|_{\mathbf{C}_{\mathrm{i}}=\text { const }}= \\
& \mu(\operatorname{det} \mathbf{C})^{-1 / 3} \mathbf{C}^{-1} \cdot\left\{\mathbb{P}:\left(\mathbf{C} \cdot \frac{\partial \mathbf{C}_{\mathrm{i}}^{-1}}{\partial \mathbf{C}}\right)\right\} .
\end{aligned}
$$

It follows from (38)-(40) that for any $\mathbf{Y} \in$ Sym

$$
\frac{\partial \mathbf{C}_{i}^{-1}}{\partial \mathbf{C}}: \mathbf{Y}=-\frac{\mu \Delta t}{\eta}\left(\boldsymbol{\Phi}^{-1}\left(\mathbf{Y C}^{-1}\right)^{\mathrm{D}} \overline{\mathbf{C}}\right)^{\mathrm{D}} \mathbf{C}_{\mathrm{i}}^{-1}
$$


Note that $\operatorname{tr}(\mathbf{A B})=\operatorname{tr}(\mathbf{B A})$ and $\operatorname{tr}\left(\mathbf{A B}^{\mathrm{D}}\right)=\operatorname{tr}\left(\mathbf{A}^{\mathrm{D}} \mathbf{B}\right)=\operatorname{tr}\left(\mathbf{A}^{\mathrm{D}} \mathbf{B}^{\mathrm{D}}\right)$ for arbitrary $\mathbf{A}, \mathbf{B}$. Using these properties, we get for any $\mathbf{X}, \mathbf{Y} \in$ Sym

$$
\begin{aligned}
& \mathbf{X}: \mathbb{T}: \mathbf{Y}= \mu(\operatorname{det} \mathbf{C})^{-1 / 3} \mathbf{X}:\left(\mathbf{C}^{-1}\left(\mathbf{C} \frac{\partial \mathbf{C}_{\mathrm{i}}^{-1}}{\partial \mathbf{C}}: \mathbf{Y}\right)^{\mathrm{D}}\right)= \\
& \mu(\operatorname{det} \mathbf{C})^{-1 / 3} \operatorname{tr}\left\{\left(\mathbf{X C}^{-1}\right)^{\mathrm{D}}\left(\mathbf{C} \frac{\partial \mathbf{C}_{\mathrm{i}}^{-1}}{\partial \mathbf{C}}: \mathbf{Y}\right)\right\} \stackrel{(41)}{=} \\
&-\frac{\mu^{2} \Delta t}{\eta}(\operatorname{det} \mathbf{C})^{-1 / 3} \operatorname{tr}\left\{\left(\mathbf{X C}^{-1}\right)^{\mathrm{D}}\left(\mathbf{C}\left(\mathbf{\Phi}^{-1}\left(\mathbf{Y C}^{-1}\right)^{\mathrm{D}} \overline{\mathbf{C}}\right)^{\mathrm{D}} \mathbf{C}_{\mathrm{i}}^{-1}\right)\right\}= \\
&-\frac{\mu^{2} \Delta t}{\eta}(\operatorname{det} \mathbf{C})^{-1 / 3} \operatorname{tr}\left\{\mathbf{C}_{\mathrm{i}}^{-1}\left(\mathbf{X} \mathbf{C}^{-1}\right)^{\mathrm{D}} \mathbf{C}\left(\mathbf{\Phi}^{-1}\left(\mathbf{Y} \mathbf{C}^{-1}\right)^{\mathrm{D}} \overline{\mathbf{C}}\right)^{\mathrm{D}}\right\}= \\
&-\frac{\mu^{2} \Delta t}{\eta}(\operatorname{det} \mathbf{C})^{-2 / 3}(\operatorname{det} \mathbf{\Phi})^{1 / 3} \operatorname{tr}\left\{\left(\mathbf{\Phi}^{-1}\left(\mathbf{X} \mathbf{C}^{-1}\right)^{\mathrm{D}} \mathbf{C}\right)^{\mathrm{D}}\left(\mathbf{\Phi}^{-1}\left(\mathbf{Y} \mathbf{C}^{-1}\right)^{\mathrm{D}} \mathbf{C}\right)^{\mathrm{D}}\right\} .
\end{aligned}
$$

Obviously, this expression is symmetric with respect to $\mathbf{X}$ and $\mathbf{Y}$. Thus, $\mathbf{X}: \mathbb{T}: \mathbf{Y}=\mathbf{Y}$ : $\mathbb{T}: \mathbf{X}$. The symmetry of the consistent tangent $\frac{\partial \tilde{\mathbf{T}}_{1}(\mathbf{C})}{\partial \mathbf{C}}$ is thus proved.

\section{Appendix E (trajectory of the exact solution)}

Consider the initial value problem (10), (11) with $\mathbf{C}(t)=$ const. Suppose that the numerical solution at time instance $t_{n}$ is given by

$$
{ }^{n} \mathbf{C}_{\mathrm{i}}=\overline{\mathbf{C}_{\mathrm{i}}^{0}+{ }^{n} \varphi \overline{\mathbf{C}}}
$$

with some suitable ${ }^{n} \varphi \geq 0$. Substituting this into the update formula (23) we get

$$
{ }^{n+1} \mathbf{C}_{\mathrm{i}}=\overline{{ }^{n} \mathbf{C}_{\mathrm{i}}+\frac{\mu \Delta t}{\eta} \overline{\mathbf{C}}}=\overline{\overline{\mathbf{C}_{\mathrm{i}}^{0}+{ }^{n} \varphi \overline{\mathbf{C}}+\frac{\mu \Delta t}{\eta} \overline{\mathbf{C}}}}=\overline{\mathbf{C}_{\mathrm{i}}^{0}+{ }^{n+1} \varphi \overline{\mathbf{C}}}
$$

with a suitable ${ }^{n+1} \varphi \geq 0$. Since the assumption (42) is satisfied for $n=1$, it is satisfied for any $n=1,2,3, \ldots$. Finally, it is known that the numerical solution converges to the exact solution as $\Delta t \rightarrow 0$. Therefore, for any $t \geq t^{0}$, we get from (42)

$$
\mathbf{C}_{\mathrm{i}}(t)=\overline{\mathbf{C}_{\mathrm{i}}^{0}+\varphi(t) \overline{\mathbf{C}}} .
$$

Thus, the trajectory of the exact solution is given by (43). By substituting this relation into (10), the following initial value problem for $\varphi$ is obtained

$$
\dot{\varphi}=\frac{\mu}{\eta}\left(\operatorname{det}\left(\mathbf{C}_{\mathrm{i}}^{0}+\varphi \overline{\mathbf{C}}\right)\right)^{1 / 3},\left.\quad \varphi\right|_{t=t^{0}}=0 .
$$


In other words, the parametrization (43) allows to reduce the six-dimensional flow rule to a one-dimensional one.

Finally, we have $\varphi \rightarrow 0$ as $t \rightarrow t^{0}$. Thus, since $\operatorname{det}\left(\mathbf{C}_{\mathrm{i}}^{0}\right)=1$ we $\operatorname{get} \operatorname{det}\left(\mathbf{C}_{\mathrm{i}}^{0}+\varphi(t) \overline{\mathbf{C}}\right) \approx 1$ as $t \rightarrow t^{0}$. Substituting this into (5), we get $\varphi(t) \approx \frac{\mu\left(t-t^{0}\right)}{\eta}$ as $t \rightarrow t^{0}$.

\section{References}

[1] C. Balan, C. Tsakmakis, A finite deformation formulation of the 3-parameter viscoelastic fluid, Journal of non-newtonian fluid mechanics, 103(1) (2002), 45-64.

[2] C. Bröcker, A. Matzenmiller, An enhanced concept of rheological models to represent nonlinear thermoviscoplasticity and its energy storage behavior, Continuum Mech. Thermodyn. DOI 10.1007/s00161-012-0268-3

[3] W. Dettmer, S. Reese, On the theoretical and numerical modelling of Armstrong-Frederick kinematic hardening in the finite strain regime, Computer Methods in Applied Mechanics and Engineering, 193 (2004) 87-116.

[4] A. Drozdov, Viscoelastic Structures: Mechanics of Growth and Aging, Academic Press, 1998

[5] B. Eidel, C. Kuhn, Order reduction in computational inelasticity: Why it happens and how to overcome it - The ODE-case of viscoelasticity, International Journal for Numerical Methods in Engineering, 87 (2011) 1046-1073.

[6] B. Eidel, F. Stumpf, J. Schröder, Finite strain viscoelasticity: how to consistently couple discretizations in time and space on quadrature-point level for full order $p \geq 2$ and a considerable speed-up, Computational Mechanics, (2013) DOI 10.1007/s00466-013-0823-1

[7] H. P. Feigenbaum, J. Dugdale, Y. F. Dafalias, K. I. Kourousis, J. Plesek, Multiaxial ratcheting with advanced kinematic and directional distortional hardening rules, International Journal of Solids and Structures, 49 (2012) 3063-3076.

[8] T. C. Gasser, C. Forsell, The numerical implementation of invariant-based viscoelastic formulations at finite strains. An anisotropic model for the passive myocardium, Computer Methods in Applied Mechanics and Engineering, 200 (2011) 3637-3645.

[9] A. N. Gent, Simple rotary dynamic testing machine. Rubber Chemistry and Technology, 34(3) (1961) 790-794.

[10] S. Govindjee, S. Reese, A Presentation and comparison of two large deformation viscoelasticity models, Journal of Engineering Materials and Technology, 119, (1997) 251255.

[11] S. Hartmann, G. Lührs, P. Haupt, An efficient stress algorithm with applications in viscoplasticity and plasticity, International Journal for Numerical Methods in Engineering, 40 (1997) 991-1013. 
[12] S. Hartmann, Computation in finite-strain viscoelasticity: finite elements based on the interpretation as differential-algebraic equations, Computer Methods in Applied Mechanics and Engineering, 191 (2002) 1439-1470.

[13] S. Hartmann, Finite-Elemente Berechnung inelastischer Kontinua. Interpretation als Algebro-Differentialgleichungssysteme, Habilitation thesis, Kassel, 2003.

[14] S. Hartmann, A.-W. Hamkar, Rosenbrock-type methods applied to finite element computations within finite strain viscoelasticity, Computer Methods in Applied Mechanics and Engineering, 199 (2010) 1455-1470.

[15] K. Hasanpour, S. Ziaei-Rad, M. Mahzoon, A large deformation framework for compressible viscoelastic materials: Constitutive equations and finite element implementation, International Journal of Plasticity, 25 (2009) 1154-1176.

[16] P. Haupt, Continuum Mechanics and Theory of Materials, 2nd edition, Springer, 2002.

[17] P. Haupt, A. Lion, On finite linear viscoelasticity of incompressible isotropic materials, Acta Mechanica 159, 87-124, 2002.

[18] D. Helm, Formgedächtnislegierungen, experimentelle Untersuchung, phänomenologische Modellierung und numerische Simulation der thermomechanischen Materialeigenschaften, Universitätsbibliothek Kassel, 2001.

[19] D. Helm, Stress computation in finite thermoviscoplasticity. International Journal of Plasticity, 22 (2006) 1699-1721.

[20] D. Helm, Thermomechanics of martensitic phase transitions in shape memory alloys I, constitutive theories for small and large deformations, J. Mech. Mater. Struct., 2(1) (2007) $87-112$.

[21] C. Hohl. Anwendung der Finite-Elemente-Methode zur Parameteridentifikation und Bauteilsimulation bei Elastomeren mit Mullins-Effekt. Düsseldorf: VDI Verlag 2007

[22] D. W. Holmes, J. G. Loughran, Numerical aspects associated with the implementation of a finite strain, elasto-viscoelastic-viscoplastic constitutive theory in principal stretches, Int. J. Numer. Meth. Engng, 83 (2010) 366-402.

[23] G.A. Holzapfel, On large strain viscoelasticity: continuum formulation and finite element applications to elastomeric structures. Int. J. Numer. Meth. Eng. 39, (1996) 3903-3926.

[24] N. Huber, C. Tsakmakis, Finite deformation viscoelasticity laws, Mechanics of Materials 32 (2000) $1-18$

[25] M. Itskov, Tensor Algebra and Tensor Analysis for Engineers: With Applications to Continuum Mechanics (Springer, 2007).

[26] M. Johlitz, H. Steeb, S. Diebels, A. Chatzouridou, J. Batal, W. Possart, Experimental and theoretical investigation of nonlinear viscoelastic polyurethane systems, J. Mater. Sci., 42 (2007) 9894-9904

[27] M. W. Johnson, D. Segalman, A model for viscoelastic fluid behavior which allows non-affine deformation. J. Non-Newtonian Fluid Mech. 2, (1977) 255-270. 
[28] R. Klauke, T. Alshuth, J. Ihlemann, Lifetime prediction of rubber products under simpleshear loads with rotary axes. In: G. Heinrich, M. Kaliske, A. Lion, S. Reese (Editors): Constitutive Models for Rubber VI, Taylor \& Francis Group, London, (2009) 235-240.

[29] R. Klauke, T. Alshuth, J. Ihlemann, Lebensdauervorhersage von technischen Gummiwerkstoffen unter einfacher Scherung mit rotierenden Achsen. Kautschuk Gummi Kunststoffe 63, (2010) 286-290.

[30] B. Kleuter, A. Menzel, P. Steinmann, Generalized parameter identification for finite viscoelasticity, Computer Methods in Applied Mechanics and Engineering, 196 (2007) 33153334.

[31] N. Koprowski-Theiß, M. Johlitz, S. Diebels, Modelling of a cellular rubber with nonlinear viscosity functions, Experimental Mechanics, 51 (2011) 749-765.

[32] E. Kröner, Allgemeine Kontinuumstheorie der Versetzungen und Eigenspannungen, Arch. Rational Mech. Anal., 4 (1959) 273-334.

[33] F. P. La Mantia, Non linear viscoelasticity of polymeric liquids interpreted by means of a stress dependence of free volume. Rheol. Acta. 16, 302-308 (1977)

[34] R. Landgraf, J. Ihlemann, Vergleich zweier Ansätze zur Beschreibung nichtlinearer Viskoelastizität auf Basis des Maxwell-Elements, PAMM, (10) 1, 303-304.

[35] E. H. Lee, Elastic-plastic deformation at finite strains, J. Appl. Mech., 36 (1969) 1-6.

[36] S. Lejeunes, A. Boukamel, S. Méo, Finite element implementation of nearly-incompressible rheological models based on multiplicative decompositions, Computers and Structures 89, (2011) 411-421.

[37] A. I. Leonov, Nonequilibrium thermodynamics and rheology of viscoelastic polymeric media. Rheol. Acta 15, (1976) 85-98.

[38] A. Lion, A physically based method to represent the thermo-mechanical behaviour of elastomers, Acta Mechanica 123, 1-25 (1997)

[39] A. Lion, Constitutive modelling in finite thermoviscoplasticity: a physical approach based on nonlinear rheological elements, International Journal of Plasticity, 16 (2000) 469-494.

[40] A. Lion, Thermomechanik von Elastomeren, Habilitation thesis, Kassel, 2000.

[41] X. N. Meng, T. A. Laursen, Energy consistent algorithms for dynamic finite deformation plasticity, Computer Methods in Applied Mechanics and Engineering, 191 (2002) 1639-1675.

[42] MSC.Software Corporation: MSC.Marc 2010, Volume A: Theory and User Information.

[43] B. Nedjar, Frameworks for finite strain viscoelastic-plasticity based on multiplicative decompositions. Part I: Continuum formulations, Comput. Methods Appl. Mech. Engrg. 191 (2002) 1541-1562.

[44] B. Nedjar, Frameworks for finite strain viscoelastic-plasticity based on multiplicative decompositions. Part II: Computational aspects, Comput. Methods Appl. Mech. Engrg. 191 (2002) 1563-1593. 
[45] P. Neff, Mathematische Analyse multiplikativer Viskoplastizität. Ph.D. Thesis TU Darmstadt. (Shaker Verlag, 2000).

[46] I. Nishiguchi, T.-L. Sham, E. Krempl, A finite deformation theory of viscoplasticity based on overstress: Part I-Constitutive Equations. Trans. ASME J Appl. Mech. 57, (1990) 548-552.

[47] I. Nishiguchi, T.-L. Sham, E. Krempl, A finite deformation theory of viscoplasticity based on overstress: Part ll-Finite element implementation and numerical experiments. Trans. ASME J Appl. Mech. 57, (1990) 553-561.

[48] G. Pearson, S. Middleman, Elongation flow behavior of viscoelastic liquids: modelling bubble dynamics with viscoelastic constitutive relations. Rheol. Acta 17, (1978) 500-510.

[49] D. Peric, A.J.L. Crook, Computational strategies for predictive geology with reference to salt tectonics, Comput. Methods Appl. Mech. Engrg. 193 (2004) 5195-5222

[50] G. Rauchs, Finite element implementation including sensitivity analysis of a simple finite strain viscoelastic constitutive law, Computers and Structures 88 (2010) 825-836

[51] S. Reese, S. Govindjee, A theory of finite viscoelasticity and numerical aspects, International Journal of Solids and Structures, 35 (1998), 3455-3482.

[52] S. Reese, Thermomechanische Modellierung gummiartiger Polymerstrukturen, Habilitation thesis, Hannover, 2000.

[53] M. Reiner, Deformation, Strain and Flow. An Elementary Introduction to Rheology, 2nd edition, 1960.

[54] A. V. Shutov, R. Kreißig, Finite strain viscoplasticity with nonlinear kinematic hardening: Phenomenological modeling and time integration, Computer Methods in Applied Mechanics and Engineering, 197, 2015-2029 (2008).

[55] A. V. Shutov, R. Kreißig, Application of a coordinate-free tensor formalism to the numerical implementation of a material model, ZAMM, 88, 11, 888-909 (2008).

[56] A. V. Shutov, R. Kreißig, Geometric integrators for multiplicative viscoplasticity: Analysis of error accumulation, Comput. Methods Appl. Mech. Engrg. 199 (2010) 700-711.

[57] A. V. Shutov, C. Kuprin, J. Ihlemann, M. F.-X.Wagner, C. Silbermann, Experimentelle Untersuchung und numerische Simulation des inkrementellen Umformverhaltens von Stahl 42CrMo4, Mat.-wiss. u.Werkstofftech., 41(9), (2010) 765-775.

[58] A. V. Shutov, S. Panhans, R. Kreißig, A phenomenological model of finite strain viscoplasticity with distortional hardening, ZAMM, 91, 8, 653-680 (2011).

[59] A. V. Shutov, S. Pfeiffer, J. Ihlemann, On the simulation of multi-stage forming processes: invariance under change of the reference configuration, Mat.-wiss. u.Werkstofftech., 43(7), (2012) 617-625.

[60] A. V. Shutov, J. Ihlemann, A viscoplasticity model with an enhanced control of the yield surface distortion, International Journal of Plasticity, 39, 152-167 (2012).

[61] J. C. Simo, G. Meschke, A new class of algorithms for classical plasticity extended to finite strains. Application to geomaterials, Computational mechanics, 11(4), (1993) 253-278. 
[62] J. C. Simo, C. Miehe, Associative coupled thermoplasticity at finite strains: formulation, numerical analysis and implementation. Computer Methods in Applied Mechanics and Engineering 98, (1992) 41-104.

[63] J. C. Simo, Algorithms for static and dynamic multiplicative plasticity that preserve the classical return mapping schemes of the infinitesimal theory. Computer Methods in Applied Mechanics and Engineering 99, (1992) 61-112.

[64] J. Simo, T. Hughes, Computational inelasticity, Springer, 1998.

[65] T. Sussman, K. J. Bathe, A finite element formulation for nonlinear incompressible elastic and inelastic analysis. Computers and Structures, 26(1), (1987) 357-409.

[66] I. Vladimirov, M. Pietryga, S. Reese, On the modelling of non-linear kinematic hardening at finite strains with application to springback - Comparison of time integration algorithms, Int. J. Numer. Meth. Engng 75 (2008), 1-28.

[67] E. Wiechert, "Ueber elastische Nachwirkung", Dissertation, Königsberg University, Germany, 1889.

[68] O. H. Yeoh, Some forms of the strain energy function for rubber, Rubber Chemistry and technology, 66(5) (1993), 754-771. 